\title{
Studies on the Regioselectivity of the Cyclization of Tryptophanol-Derived Oxazolopiperidone Lactams
}

\author{
Mercedes Amat, ${ }^{*[\mathrm{a}]}$ Núria Llor, ${ }^{[\mathrm{a}]}$ Fabiana Subrizi, ${ }^{[\mathrm{a}]}$ Maria Pérez, ${ }^{[\mathrm{a}]}$ Elies Molins, ${ }^{[\mathrm{b}]}$ and \\ Joan Bosch ${ }^{[a]}$
}

Keywords: Lactams / Asymmetric synthesis / Cyclization / Bischler-Napieralski / $\alpha$-Amidoalkylation

Cyclization of the lactam carbonyl on the indole ring in tryptophanol-derived oxazolopiperidone lactams $\mathbf{2}$ and $\mathbf{6}$, under the classical $\mathrm{POCl}_{3}$-promoted Bischler-Napieralski conditions and under neutral conditions via the corresponding thiolactam, is studied. Whereas tricyclic lactam 2 only leads to products coming from an $\alpha$-amidoalkylation process, bicyclic lactam $\mathbf{6}$ undergoes cyclization on the lactam carbonyl, leading to the expected indolo[2,3- $a]$ quinolizidine derivatives.

\section{Introduction}

Tryptophanol-derived oxazolopiperidone lactams have proven to be versatile chiral building blocks for the enantioselective synthesis of indole alkaloids. ${ }^{[1]}$ These lactams are easily accessible in enantiopure form in a single synthetic step by a stereoselective cyclocondensation reaction between $(S)$-tryptophanol and an appropriate $\delta$-oxo acid derivative. ${ }^{[2]}$ Tryptophanol not only constitutes the source of chirality but can also be used in subsequent steps to assemble complex polycyclic targets by regioand stereocontrolled cyclization reactions on the indole ring.

Taking advantage of the functionalization present in the oxazolopiperidone lactam, an electrophilic cyclization on the indole 2-position can involve either the hemiaminal ether carbon via an $N$-acyliminium cation (via a $)^{[1 \mathrm{a}-\mathrm{d}, 2]}$ or the lactam carbonyl via a Bischler-Napieralski-type reaction (via b) ${ }^{[1 \mathrm{e}, 3]}$ leading to regioisomeric indolo[2,3- $a$ ]quinolizidines (when $\mathrm{R}_{1} \neq \mathrm{H}$ ). Additionally, by choosing the appropriate reaction conditions, the intramolecular $\alpha$-amidoalkylation allows the stereocontrolled generation of $\mathrm{C}-12 \mathrm{~b}$ epimeric derivatives. On the other hand, a Lewis acid/Et $\mathrm{SiH}_{3} \mathrm{SiH}$-promoted cyclization on the indole 3-position from $N_{a}$-tosyl derivatives provides straightforward access to the spiro[indole-3,1'-indolizidine] framework $^{[4]}$ present in a large number of alkaloids (via c). These complementary types of cyclization are shown in Scheme 1.

[a] Laboratory of Organic Chemistry, Faculty of Pharmacy, and Institute of Biomedicine (IBUB), University of Barcelona, Av. Joan XXIII s/n, 08028 Barcelona, Spain

Fax: (+34) 934-024-539

E-mail: amat@ub.edu

[b] Institut de Ciència de Materials de Barcelona (CSIC), Campus UAB, 0 8193 Cerdanyola, Spain

Supporting information for this article is available on the WWW under http://dx.doi.org/10.1002/ejoc.Xxxxxxxxx

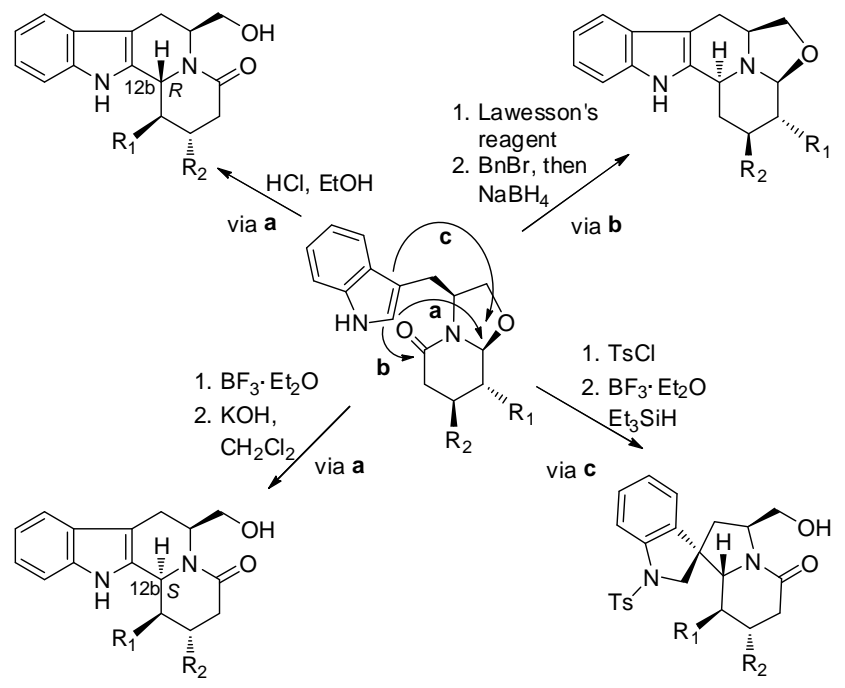

Scheme 1. Regio- and stereocontrolled cyclizations from $(S)$-tryptophanolderived oxazolopiperidone lactams.

Although there are many examples of acid-promoted intramolecular $\alpha$-amidoalkylation reactions from $(S)$-tryptophanolderived oxazolopiperidone lactams, cyclizations involving the lactam carbonyl have been little explored. In fact, our initial attempts to perform a Bischler-Napieralski cyclization under the classical conditions $\left(\mathrm{POCl}_{3}\right.$, then $\left.\mathrm{NaBH}_{4}\right)$ were unsuccessful ${ }^{[1 \mathrm{e}, 5]}$ due to the tendency of these lactams to undergo acid-promoted intramolecular $\alpha$-amidoalkylation reactions. However, the desired cyclization on the lactam carbonyl can be satisfactorily performed under non-acidic conditions by a modified Bischler-Napieralski procedure via a (benzylthio)iminium intermediate. ${ }^{[1 \mathrm{e}]}$ In the only example reported to date of a successful $\mathrm{POCl}_{3}$-promoted Bischler-Napieralski cyclization from a tryptophanol-derived oxazolopiperidone lactam, ${ }^{[3]}$ the hemiaminal ether carbon incorporates an additional substituent, which, for steric reasons, seems to hamper the intramolecular $\alpha$-amidoalkylation reaction. 


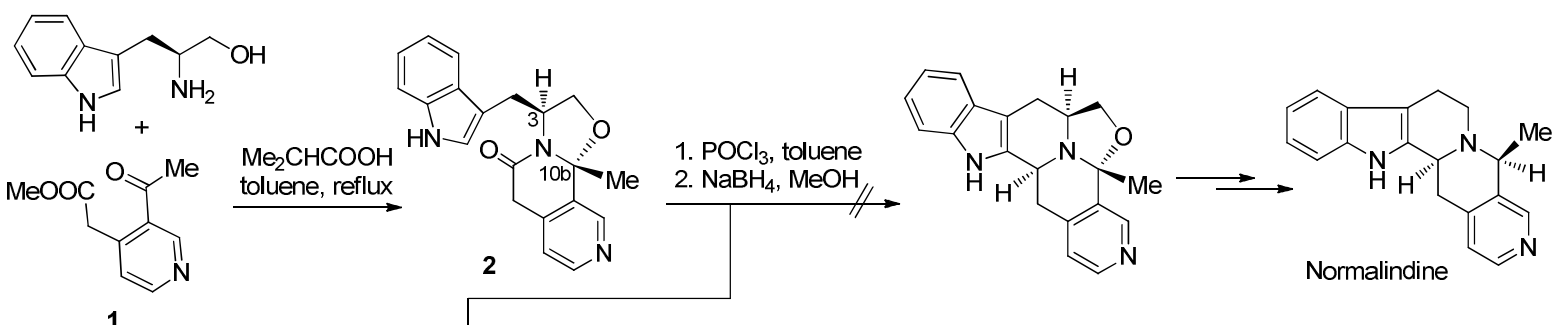

1

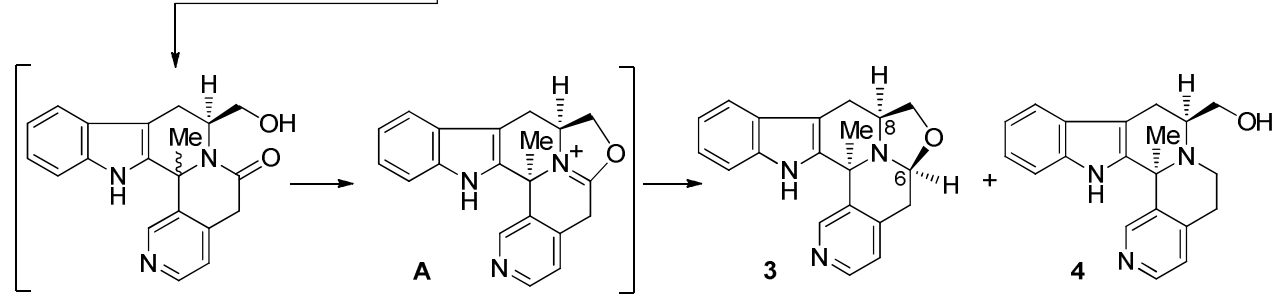

Scheme 2. Studies on the synthesis of normalindine. Cyclization under Bischler-Napieralski conditions.

\section{Results and Discussion}

With these precedents in mind, we decided to explore if the methodology could be applied to the synthesis of malindine alkaloids, for instance normalindine ${ }^{[6]}$ which are characterized by the presence of a partially reduced 1-methyl-2,7-naphthyridine moiety (Scheme 2). The synthesis would involve as the key steps a cyclocondensation reaction between $(S)$-tryptophanol and pyridine $\delta$-keto ester 1, a Bischler-Napieralski cyclization from the resulting tricyclic lactam, and finally, the reductive opening of the oxazolidine ring with subsequent removal of the hydroxymethyl appendage. ${ }^{[7]}$ The quaternary carbon $10 \mathrm{~b}$ on the oxazolidine ring would hamper the $\alpha$-amidoalkylation process in favor of the Bischler-Napieralski cyclization.

As foreseen, the cyclocondensation reaction took place in acceptable yield $(65 \%)$ and excellent stereoselectivity to give the trans 3-H/10b-Me 2,7-naphthyridine lactam $2 .{ }^{[8]}$ However, rather unexpectedly, treatment of this lactam under classical Bischler-Napieralski conditions $\left(\mathrm{POCl}_{3}\right.$, then $\left.\mathrm{NaBH}_{4}\right)$ resulted in an $\alpha$-amidoalkylation process, leading to hexacycle 3 ( $42 \%$ yield) and pentacycle 4 (21\% yield). No products coming from the cyclization of the lactam carbonyl on the indole ring were detected.

The formation of $\mathbf{3}$ can be rationalized by considering a rapid generation of an $\mathrm{N}$-acyliminium cation ${ }^{[9]}$ and its cyclization on the indole ring, a subsequent interaction of the hydroxymethyl substituent with the chloroiminium intermediate generated from the lactam carbonyl, and a final reduction by $\mathrm{NaBH}_{4}$ of the resulting oxazolinium salt. ${ }^{[10]}$ A further reduction, with opening of the oxazolidine ring, would lead to 4.
The cis $1-\mathrm{Me} / 3-\mathrm{H}$ relative stereochemistry for the tetrahydro- $\beta$ carboline moiety, established by NOE experiments, probably results from an acid-promoted epimerization ${ }^{[11]}$ of the quaternary stereocenter initially formed in the cyclization, ${ }^{[12]}$ driven by the generation of a cis oxazolinium intermediate, A. A final stereoelectronically controlled ${ }^{[13]}$ axial attack of the hydride installs the all-cis relative configuration of $\mathbf{3}$.

To avoid the undesirable acid-promoted $\alpha$-amidoalkylation reaction, we decided to perform a Bischler-Napieralski-type cyclization under neutral conditions ${ }^{[1 \mathrm{e}]}$ from the thiolactam derived from 2. Surprisingly, treatment of lactam 2 with Lawesson's reagent led in excellent yield $(81 \%)$ to the hexacyclic thioderivative 5 instead of the expected thiolactam (Scheme 3). The formation of $\mathbf{5}$ can be accounted for by considering that, once the thiolactam was formed, the generation of the $N$-thioacyl iminium ion $\mathbf{B}$ was facilitated by the lower electronegativity of the sulfur atom. Cyclization on the indole ring would afford pentacyclic intermediate C. A subsequent cyclization via the $O$ alkyl phenylphosphonodithioic acid intermediate $\mathbf{D}^{[14]}$ would lead to 5 .

The above results indicate that an acidic environment is not the only factor to favor the competitive $\alpha$-amidoalkylation reaction. Most probably, due to the presence of the fused $\pi$-deficient pyridine, the benzylic character of the $N$-acyl (or thioacyl) iminium species generated by the opening of the oxazolidine ring also plays an important role.

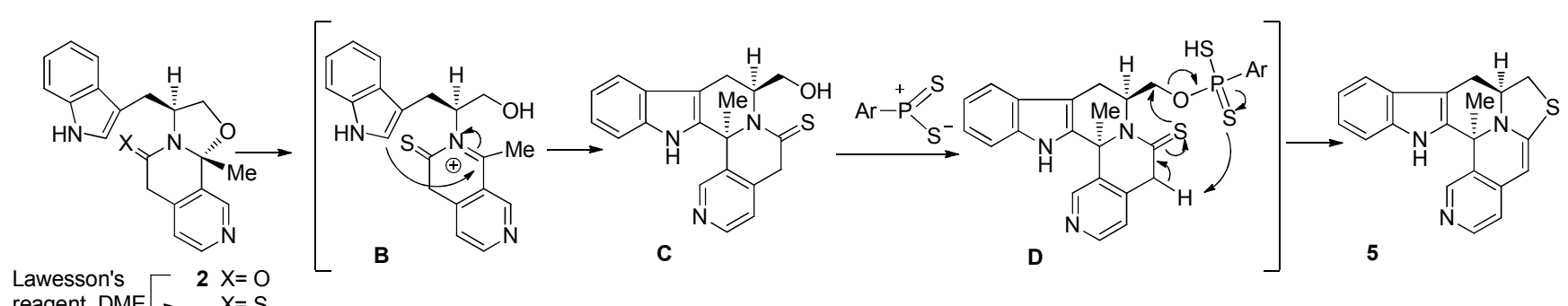

Scheme 3. Reaction of lactam 2 with Lawesson's reagent. 
To gain further insight into the factors governing the regioselectivity of the cyclization on the carbonyl group of tryptophanol-derived oxazolopiperidone lactams, we decided to study the Bischler-Napieralski cyclization from a model lactam $\mathbf{6}$, which lacks the pyridine ring present in 2. This lactam was prepared by cyclocondensation between $(S)$-tryptophanol and 5oxohexanoic acid. Under the usual reaction conditions (toluene, reflux), a mixture of 6 and its 8a-epimer (80\% yield, 87:13 ratio) was obtained $^{[15]}$ (Scheme 4). A more convenient experimental procedure involved the use of microwaves: ${ }^{[16]}$ irradiation of an equimolecular mixture of the starting materials for $10 \mathrm{~min}$ at 110 ${ }^{\circ} \mathrm{C}$ provided a 92:8 diastereoisomeric mixture of $\mathbf{6}$ and its $8 \mathrm{a}-$ epimer in $77 \%$ yield, with only traces of the cyclized product 9 (Scheme 5) being detected.

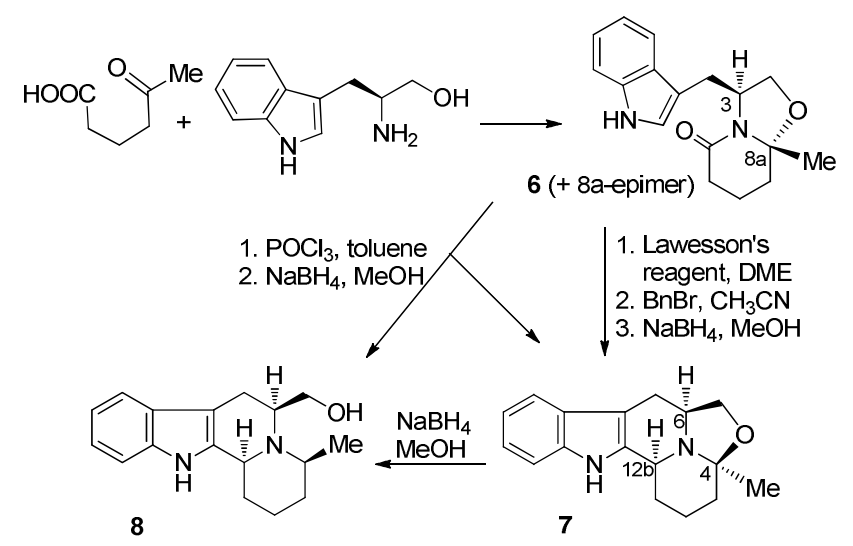

Scheme 4. Cyclizations on the lactam carbonyl from lactams 6

As expected, cyclization on the lactam carbonyl took place satisfactorily from lactams $\mathbf{6}$, not only under neutral conditions via a thiolactam but also under the classical $\mathrm{POCl}_{3}$-promoted Bischler-Napieralski conditions. Thus, the epimeric mixture of $\mathbf{6}$ was converted to the corresponding thiolactams (54\%), which were treated with benzyl bromide and then $\mathrm{NaBH}_{4}$, to regio- and stereoselectively give (51\%) a single pentacyclic indoloquinolizidine derivative 7. Similarly, treatment of the epimeric mixture of $\mathbf{6}$ with $\mathrm{POCl}_{3}$ and then $\mathrm{NaBH}_{4}$ stereoselectively led to a $3: 2$ mixture of pentacycle $\mathbf{7}$ and indoloquinolizidine 8 in $52 \%$ overall yield. ${ }^{[17]}$ The former was quantitatively converted to $\mathbf{8}$ by additional treatment with $\mathrm{NaBH}_{4}{ }^{[18]}$

The all-cis stereochemistry of 7, established by NOE experiments (4-Me/6-H, 4-Me/12b-H, and 6-H/12b-H), deserves some comment as the sterocenters bearing the methyl substituent in the major starting lactam 6 and in the cyclized product 7 have the opposite relative configuration. This indicates that during the above cyclization an equilibration has occurred via an open oxazolidine intermediate, ultimately leading to the less strained cis 4-Me/6- $\mathrm{H}$ iminium salt $\mathbf{E}$ (Figure 1), which undergoes a final stereoelectronically controlled ${ }^{[13]}$ axial attack of the hydride. Finally, also worthy of comment is the inversion of the configuration during the reductive opening of the oxazolidine ring to give $\mathbf{8}$, once again involving a stereoelectronically controlled attack of the hydride on the less hindered face of the intermediate iminium species $\mathbf{F}$.
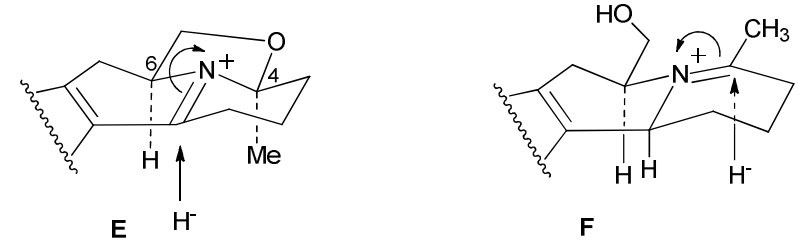

Figure 1. Stereocontrolled reduction of iminium ion intermediates.

The above successful Bischler-Napieralski-type cyclizations make evident that the intramolecular $\alpha$-amidoalkylation process is slowed down by the presence of the additional substituent at the 2position of the oxazolidine ring. In fact, lactams $\mathbf{6}$ were recovered unchanged (only trace amounts of the cyclized product 9 were detected) after treatment with $\mathrm{HCl}(0.6 \mathrm{M}$ in EtOH, rt, $24 \mathrm{~h})$, whereas these reaction conditions promote a clean and highly stereoselective $\alpha$-amidoalkylation (95\% yield) when applied to the corresponding lactams lacking the angular methyl substituent. ${ }^{[5]}$ Under more drastic conditions (1.25 M HCl in $\mathrm{EtOH}, 70{ }^{\circ} \mathrm{C}, 72 \mathrm{~h}$ ), cyclization of 6 took place in good yield $(70 \%)$ but with a low stereoselectivity to give a nearly 1:1 mixture of trans and cis indoloquinolizidines $\mathbf{1 0}$ and $12 \mathrm{~b}$-epi-9, probably because the strong acidity causes the epimerization at $\mathrm{C}-12 \mathrm{~b}$ of the indolo[2,3$a$ ]quinolizidine system $^{[12]}$ (Scheme 5). From the synthetic standpoint, the best results were obtained using a $0.9 \mathrm{M}$ solution of TFA in $\mathrm{CH}_{2} \mathrm{Cl}_{2}(\mathrm{rt}, 20 \mathrm{~h})$, since a $4: 1$ stereoisomeric mixture of indoloquinolizidines 9 and 12b-epi-9 was obtained in $86 \%$ yield. The absolute configuration of the cyclized products 9 and 12b-epi9 was unambiguously determined by X-ray crystallographic analysis. ${ }^{[19]}$

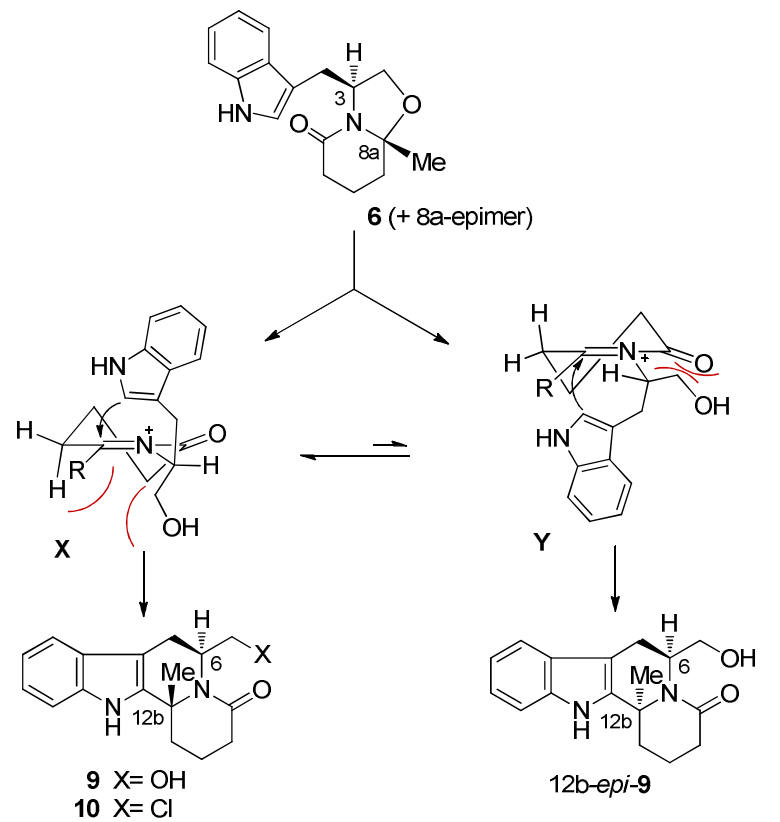

Scheme 5. $\alpha$-Amidoalkylation reaction from lactams 6.

The stereoselective formation of 9 leading to a trans 6-H/12bMe stereochemistry can be rationalized by analyzing the two 
possible reactive chair-like conformations ( $\mathbf{X}$ and $\mathbf{Y} ; \mathrm{R}=\mathrm{Me}$ ) of the intermediate $N$-acyliminium cation. ${ }^{[9]}$ Cyclization takes place faster from conformation $\mathbf{X}$, which avoids the severe $\mathrm{A}^{(1,3)}$ strain between the hydroxymethyl substituent and the lactam carbonyl group in the transition state. ${ }^{[2]}$ The lower stereoselectivity in the cyclization of 6 compared with that of the corresponding demethyl lactam ${ }^{[2 \mathrm{a}, 5]}$ can be explained by the stronger $\mathrm{A}^{(1,3)} \mathrm{CH}_{2} \mathrm{OH} / \mathrm{R}$ strain present in the conformation $\mathbf{X}$ when $\mathrm{R}=\mathrm{Me}$ than when $\mathrm{R}=\mathrm{H}$.

\section{Conclusions}

Although under the classical $\mathrm{POCl}_{3}$-promoted Bischler-Napieralski reaction conditions tryptophanol-derived oxazolopiperidone lactams unsubstituted at the hemiaminal ether carbon (i.e. generated from aldehyde-esters) undergo an intramolecular $\alpha$-amidoalkylation reaction instead of the expected cyclization on the lactam carbonyl, lactams substituted at this position (e.g. 6, derived from a ketone-ester) successfully undergo a regioselective Bischler-Napieralski cyclization on the lactam carbonyl, avoiding the competitive $\alpha$-amidoalkylation process.

The unexpected behavior of tricyclic lactam 2, which exclusively leads to products coming from an $\alpha$-amidoalkylation process, is a consequence of the presence of the fused $\pi$-deficient pyridine ring that originates a reactive benzylic-type $\mathrm{N}$-acyl (or thioacyl) iminium ion.

\section{Experimental Section}

General: Flash chromatography was carried out on $\mathrm{SiO}_{2}$ (silica gel 60 , SDS, 35-70 $\mu \mathrm{m}$ or using Biotage ${ }^{\circledR}$ SNAP cartridges, $50 \mu \mathrm{m}$ ). Optical rotations were measured on Perkin-Elmer 241 polarimeter. High resolution mass spectra (HMRS; LC/MSD TOF Agilent Technologies) were performed by Centres Científics i Tecnològics de la Universitat de Barcelona. Microanalyses (Carlo Erba 1106 analyzer) were performed by Centre d'Investigació i Desenvolupament (CSIC), Barcelona. Only noteworthy IR absorptions $\left(\mathrm{cm}^{-1}\right.$; Nicolet Avantar 320 FT-IR) are listed. NMR spectra were recorded at $400 \mathrm{MHz}\left({ }^{1} \mathrm{H}\right)$ and 75.4 or $100.6 \mathrm{MHz}$ $\left({ }^{13} \mathrm{C}\right)$.

(3S,10bR)-3-(3-Indolylmethyl)-5-oxo-2,3,6,10b-tetrahydro-5Hoxazolo[2,3-a][2,7]naphthyridine (2): (S)-tryptophanol (228 mg, $1.2 \mathrm{mmol})$ and isobutyric acid $(132 \mathrm{mg}, 1.5 \mathrm{mmol})$ were added to a solution of compound $\mathbf{1}^{[21]}(200 \mathrm{mg}, 1 \mathrm{mmol})$ in toluene $(16 \mathrm{~mL})$. The mixture was heated at reflux for $19 \mathrm{~h}$ with azeotropic elimination of water by a Dean-Stark system. The resulting mixture was cooled and concentrated under reduced pressure. The residue was dissolved in $\mathrm{CH}_{2} \mathrm{Cl}_{2}$ and the solution was washed twice with saturated aqueous $\mathrm{NaHCO}_{3}$ solution. The organic phase was dried, filtered, and concentrated. Flash chromatography (2:8 hexane/EtOAc) gave lactams 2 and 10b-epi-2 as a 96:4 mixture (218 mg, 65\%).

Data for 2 (from the mixture): ${ }^{1} \mathrm{H}$ NMR (400 MHz, $\mathrm{CDCl}_{3}, \mathrm{COSY}$, HSQC, $\left.25^{\circ} \mathrm{C}\right): \delta=1.52\left(\mathrm{~s}, 3 \mathrm{H}, \mathrm{CH}_{3}\right), 3.16(\mathrm{dd}, J=14.0,10.0 \mathrm{~Hz}$, $\left.1 \mathrm{H}, \mathrm{CH}_{2}\right), 3.50\left(\mathrm{dd}, J=14.0,3.6 \mathrm{~Hz}, 1 \mathrm{H}, \mathrm{CH}_{2}\right), 3.58(\mathrm{~d}, J=18.4$ $\mathrm{Hz}, 1 \mathrm{H}, \mathrm{H}-6$ ), 3.73 (d, $J=18.4 \mathrm{~Hz}, 1 \mathrm{H}, \mathrm{H}-6$ ), 3.93 (dd, $J=8.6,6.4$ $\mathrm{Hz}, 1 \mathrm{H}, \mathrm{H}-2), 4.09$ (dd, $J=8.6,4.8 \mathrm{~Hz}, 1 \mathrm{H}, \mathrm{H}-2), 4.60$ (m, 1H, H-
3), 7.11 (d, $J=2.0 \mathrm{~Hz}, 1 \mathrm{H}, \mathrm{H}-2$ '), 7.15 (masked d, 1H, H-7), 7.16 (td, $J=8.0,1.2 \mathrm{~Hz}, 1 \mathrm{H}, \mathrm{H}-6$ '), 7.21 (td, $J=7.2,1.2 \mathrm{~Hz}, 1 \mathrm{H}, \mathrm{H}-5$ '), 7.37 (d, $\left.J=8.0 \mathrm{~Hz}, 1 \mathrm{H}, \mathrm{H}-7^{\prime}\right), 7.80$ (d, $J=7.2 \mathrm{~Hz}, 1 \mathrm{H}, \mathrm{H}-4$ '), 8.32 (br. s, 1H, NH), 8.52 (br. s, 1H, H-8), 8.69 (br. s, 1H, H-10) ppm. ${ }^{13} \mathrm{C} \mathrm{NMR}\left(100.6 \mathrm{MHz}, \mathrm{CDCl}_{3}, 25{ }^{\circ} \mathrm{C}\right): \delta=28.2\left(\mathrm{CH}_{3}\right), 28.5\left(\mathrm{CH}_{2}\right)$, 37.6 (C-6), 56.5 (C-3), 69.1 (C-2), 92.8 (C-10b), 111.2 (C-7'), 111.6 (C-3a'), 119.1 (C-4'), 119.8 (C-6'), 121.9 (C-7), 122.3 (C5'), 122.5 (C-2'), 127.6 (C-7a'), 136.2 (C-10a), 139.1 (C-6a), 144.7 (C-10), 149.4 (C-8), 165.6 (CO) ppm. IR (NaCl): v = 1411, 1651, $3292 \mathrm{~cm}^{-1}$. HRMS: calcd. for $\mathrm{C}_{20} \mathrm{H}_{20} \mathrm{~N}_{3} \mathrm{O}_{2}[\mathrm{M}+\mathrm{H}]^{+}$: 334.1550; found: 334.1552 .

Data for 10b-epi-2 (from an enriched mixture): ${ }^{1} \mathrm{H}$ NMR (400 $\mathrm{MHz}, \mathrm{CDCl}_{3}, 25{ }^{\circ} \mathrm{C}$ ): $\delta=1.54\left(\mathrm{~s}, 3 \mathrm{H}, \mathrm{CH}_{3}\right), 2.63$ (dd, $J=14.0$, $\left.10.0 \mathrm{~Hz}, 1 \mathrm{H}, \mathrm{CH}_{2}\right), 3.61\left(\mathrm{dd}, J=14.0,2.8 \mathrm{~Hz}, 1 \mathrm{H}, \mathrm{CH}_{2}\right), 3.62(\mathrm{~d}, J$ $=19.2 \mathrm{~Hz}, 1 \mathrm{H}, \mathrm{H}-6), 3.72(\mathrm{~d}, J=19.2 \mathrm{~Hz}, 1 \mathrm{H}, \mathrm{H}-6), 4.20$ (dd, $J=$ 9.6, $2.0 \mathrm{~Hz}, 1 \mathrm{H}, \mathrm{H}-2), 4.24$ (dd, $J=9.6,1.2 \mathrm{~Hz}, 1 \mathrm{H}, \mathrm{H}-2), 4.49$ (dddd, $J=10.0,5.2,2.8,2.8 \mathrm{~Hz}, 1 \mathrm{H}, \mathrm{H}-3$ ), 6.97 (d, $J=2.0 \mathrm{~Hz}, 1 \mathrm{H}$, H-2'), 7.10-7.22 (masked m, 3H, H-7, H-5', H-6'), 7.29 (br. d, $J=$ $8.0 \mathrm{~Hz}, 1 \mathrm{H}, \mathrm{H}-7^{\prime}$ ), 7.75 (d, $J=7.6 \mathrm{~Hz}, 1 \mathrm{H}, \mathrm{H}-4^{\prime}$ ), 8.16 (br. s, $1 \mathrm{H}$, $\mathrm{NH}$ ), 8.52 (masked d, 1H, H-8), 8.65 (br. s, 1H, H-10) ppm. NOESY 1D: positive NOE effect: 3-H/10b-Me.

Treatment of 2 under Bischler-Napieralski conditions: $\mathrm{POCl}_{3}$ ( $373 \mu \mathrm{L}, 4.08 \mathrm{mmol}$ ) was added to a solution of lactams 2 and epi$2(100 \mathrm{mg}, 0.3 \mathrm{mmol})$ in toluene $(3.8 \mathrm{~mL})$, and the mixture has heated at $100{ }^{\circ} \mathrm{C}$ for $1.5 \mathrm{~h}$. After cooling, the solvent was removed and the resulting brown residue was dissolved in anhydrous $\mathrm{MeOH}$ $(6 \mathrm{~mL})$. Then, $\mathrm{NaBH}_{4}(136 \mathrm{mg}, 3.6 \mathrm{mmol})$ was slowly added to the solution at $0{ }^{\circ} \mathrm{C}$, and the mixture was allowed to reach room temperature for $2 \mathrm{~h}$ and refluxed for $2.5 \mathrm{~h}$. The mixture was cooled at $0{ }^{\circ} \mathrm{C}$ and quenched by addition of saturated aqueous $\mathrm{NaHCO}_{3}$. The solvent was evaporated, and the resulting residue was dissolved in $\mathrm{CH}_{2} \mathrm{Cl}_{2}$. The organic phase was washed with brine, dried, and concentrated. Flash chromatography of the residue (95:5 $\left.\mathrm{CH}_{2} \mathrm{Cl}_{2} / \mathrm{MeOH}\right)$ afforded hexacycle $3(40 \mathrm{mg}, 42 \%)$ and pentacycle $4(20 \mathrm{mg}, 21 \%)$.

Data for 3: Yellow oil. $[\alpha]^{22}{ }_{\mathrm{D}}=-101.5\left(c=0.9, \mathrm{CHCl}_{3}\right) .{ }^{1} \mathrm{H}$ NMR (400 MHz, $\mathrm{CDCl}_{3}, \mathrm{COSY}$, HSQC, $25{ }^{\circ} \mathrm{C}$ ): $\delta=2.02\left(\mathrm{~s}, 3 \mathrm{H}, \mathrm{CH}_{3}\right.$ ), 2.90 (ddd, $J=15.2,9.2,0.8 \mathrm{~Hz}, 1 \mathrm{H}, \mathrm{H}-5), 2.97$ (dd, $J=16.5,7.6$ $\mathrm{Hz}, 1 \mathrm{H}, \mathrm{H}-9$ ), 3.04 (dd, $J=16.5,9.2 \mathrm{~Hz}, 1 \mathrm{H}, \mathrm{H}-9$ ), 3.13 (dd, $J=$ $15.2,3.2 \mathrm{~Hz}, 1 \mathrm{H}, \mathrm{H}-5), 4.02\left(\mathrm{~d}, J=6.8 \mathrm{~Hz}, 1 \mathrm{H}, \mathrm{CH}_{2} \mathrm{O}\right), 4.22(\mathrm{~m}$, $1 \mathrm{H}, \mathrm{H}-8), 4.29$ (dd, $J=6.8,4.8 \mathrm{~Hz}, 1 \mathrm{H}, \mathrm{CH}_{2} \mathrm{O}$ ), 4.57 (dd, $J=9.2$, $3.2 \mathrm{~Hz}, 1 \mathrm{H}, \mathrm{H}-6$ ), 7.10 (masked d, 1H, H-4), 7.10 (masked td, 1H, $\mathrm{H}-11), 7.15$ (td, $J=7.2,1.2 \mathrm{~Hz}, 1 \mathrm{H}, \mathrm{H}-12), 7.30$ (d, $J=8.0 \mathrm{~Hz}, 1 \mathrm{H}$, H-10), 7.47 (d, $J=7.2 \mathrm{~Hz}, 1 \mathrm{H}, \mathrm{H}-13$ ), 8.37 (d, $J=4.8 \mathrm{~Hz}, 1 \mathrm{H}, \mathrm{H}-$ 3), 8.95 (br. s, 1H, NH), 9.19 (s, 1H, H-1) ppm. NOESY 1D: positive NOE effect: $1-\mathrm{Me} / 3-\mathrm{H} .{ }^{13} \mathrm{C}$ NMR $\left(100.6 \mathrm{MHz}, \mathrm{CDCl}_{3}, 25\right.$ $\left.{ }^{\circ} \mathrm{C}\right): \delta=21.9(\mathrm{C}-9), 31.7\left(\mathrm{CH}_{3}\right), 36.3(\mathrm{C}-5), 50.9(\mathrm{C}-8), 56.9$ (C14b), $72.3\left(\mathrm{CH}_{2} \mathrm{O}\right), 83.1$ (C-6), 104.7 (9a), 111.2 (C-13), 118.2 (C10), 119.7 (C-11), 122.2 (C-12), 125.4 (C-4), 127.2 (C-9b), 135.8 (C-14a), 135.9 (C-13a), 136.5 (C-14c), 142.3 (C-1), 147.4 (C-3), $147.6(\mathrm{C}-4 \mathrm{a}) \mathrm{ppm}$. IR $(\mathrm{NaCl}): v=2942,3182 \mathrm{~cm}^{-1}$. HRMS calcd. for $\mathrm{C}_{20} \mathrm{H}_{20} \mathrm{~N}_{3} \mathrm{O}[\mathrm{M}+\mathrm{H}]^{+}$: 318.1601 ; found: 318.1601 .

Data for 4: Yellow oil. $[\alpha]^{22}{ }_{D}=-1.5(c=1.0, \mathrm{MeOH}) .{ }^{1} \mathrm{H}$ NMR (400 MHz, $\mathrm{CDCl}_{3}, \mathrm{COSY}$, HSQC, $25{ }^{\circ} \mathrm{C}$ ): $\delta=2.03$ (s, $3 \mathrm{H}, \mathrm{CH}_{3}$ ), $2.63(\mathrm{~d}, J=8.8 \mathrm{~Hz}, 1 \mathrm{H}, \mathrm{H}-9), 2.64$ (masked, 1H, H-9), 2.67-2.69 (m, 1H, H-5), 2.72 (dd, $J=9.6,3.2 \mathrm{~Hz}, 1 \mathrm{H}, \mathrm{H}-6), 3.00-3.14$ (m, 2H, 
H-5, H-6), 3.79 (dd, $J=10.8,5.2 \mathrm{~Hz}, 1 \mathrm{H}, \mathrm{CH}_{2} \mathrm{OH}$ ), 3.87 (t, $J=$ $10.8 \mathrm{~Hz}, 1 \mathrm{H}, \mathrm{CH}_{2} \mathrm{OH}$ ), $4.06(\mathrm{~m}, 1 \mathrm{H}, \mathrm{H}-8), 7.06$ (masked d, $J=4.8$ $\mathrm{Hz}, 1 \mathrm{H}, \mathrm{H}-4), 7.09$ (td, $J=7.2,1.2 \mathrm{~Hz}, 1 \mathrm{H}, \mathrm{H}-11), 7.14$ (td, $J=7.2$, $1.2 \mathrm{~Hz}, 1 \mathrm{H}, \mathrm{H}-12), 7.29$ (d, $J=7.2 \mathrm{~Hz}, 1 \mathrm{H}, \mathrm{H}-10), 7.45$ (d, $J=7.2$ $\mathrm{Hz}, 1 \mathrm{H}, \mathrm{H}-13), 8.36$ (d, $J=5.2 \mathrm{~Hz}, 2 \mathrm{H}, \mathrm{H}-3), 9.08$ (s, 1H, H-1) ppm. NOESY 1D: positive NOE effect: $1-\mathrm{Me} / 3-\mathrm{H} .{ }^{13} \mathrm{C}$ NMR (100.6 MHz, $\left.\mathrm{CDCl}_{3}, 25^{\circ} \mathrm{C}\right): \delta=19.6$ (C-9), $29.6(\mathrm{C}-5), 29.9\left(\mathrm{CH}_{3}\right)$, 36.2 (C-6), 53.9 (C-8), 58.7 (C-14b), $61.4\left(\mathrm{CH}_{2} \mathrm{O}\right), 105.9$ (C-9a), 111.2 (C-13), 118.2 (C-10), 119.7 (C-11), 122.1 (C-12), 124.2 (C4), 127.1 (C-9b), 136.2 (C-13a), 136.8 (C-14a), 137.1 (C-14c), 144.5 (C-4a), 147.3 (C-3), 147.8 (C-1) ppm. IR (NaCl): v = 1600, $3273 \mathrm{~cm}^{-1}$. HRMS calcd. for $\mathrm{C}_{20} \mathrm{H}_{22} \mathrm{~N}_{3} \mathrm{O}[\mathrm{M}+\mathrm{H}]^{+}$: 320.1757 ; found: 320.1763 .

Treatment of 2 with Lawesson's Reagent: Lawesson's reagent (210 $\mathrm{mg}, 0.52 \mathrm{mmol}$ ) was added to a solution of lactams 2 and 10bepi-2 (150 mg, $0.45 \mathrm{mmol})$ in DME $(2.7 \mathrm{~mL})$. The resulting mixture was heated at reflux for $12 \mathrm{~h}$, cooled, and concentrated. The resulting dark orange residue was dissolved in $\mathrm{CH}_{2} \mathrm{Cl}_{2}$, and the organic solution was washed twice with saturated aqueous $\mathrm{NaHCO}_{3}$ solution. The organic layer was dried, filtered, and concentrated. Flash chromatography (1:4 hexane/EtOAc) afforded $5(120 \mathrm{mg}, 81 \%)$ as a yellow oil: $[\alpha]^{22}{ }_{\mathrm{D}}=-1.8\left(c=0.5, \mathrm{CHCl}_{3}\right) .{ }^{1} \mathrm{H}$ NMR (400 MHz, $\left.\mathrm{CDCl}_{3}, \mathrm{COSY}, \mathrm{HSQC}, 25^{\circ} \mathrm{C}\right): \delta=1.69$ (s, 3H, $\mathrm{CH}_{3}$ ), 2.91 (dd, $\left.J=13.5,10.5 \mathrm{~Hz}, 1 \mathrm{H}, \mathrm{CH}_{2} \mathrm{~S}\right), 3.08$ (m, 2H, H-9), $3.45\left(\mathrm{dd}, J=13.5,6.0 \mathrm{~Hz}, 1 \mathrm{H}, \mathrm{CH}_{2} \mathrm{~S}\right), 4.39(\mathrm{~m}, 1 \mathrm{H}, \mathrm{H}-8), 5.46(\mathrm{~s}$, $1 \mathrm{H}, \mathrm{H}-5), 6.67$ (d, $J=4.8 \mathrm{~Hz}, 1 \mathrm{H}, \mathrm{H}-4), 7.09$ (m, 2H, H-11 and $\mathrm{H}-$ 12), 7.41 (d, $J=7.6 \mathrm{~Hz}, 1 \mathrm{H}, \mathrm{H}-13), 7.49$ (d, $J=7.6 \mathrm{~Hz}, 1 \mathrm{H}, \mathrm{H}-10)$, 8.08 (d, $J=4.4 \mathrm{~Hz}, 1 \mathrm{H}, \mathrm{H}-3$ ), 8.98 (s, 1H, H-1), 11.82 (br. s, $1 \mathrm{H}$, $\mathrm{NH})$ ppm. NOESY 1D: positive NOE effect: 8 -H/14b-Me. ${ }^{13} \mathrm{C}$ NMR (75.4 MHz, $\left.\mathrm{CDCl}_{3}, 25{ }^{\circ} \mathrm{C}\right): \delta=23.6\left(\mathrm{CH}_{3}\right), 27.3(\mathrm{C}-9), 33.7$ $\left(\mathrm{CH}_{2} \mathrm{~S}\right), 59.9$ (C-14b), 60.3 (C-8), 90.6 (C-5), 106.9 (9a), 111.9 (C13), 115.8 (C-4), 117.8 (C-10), 119.2 (C-11), 121.9 (C-12), 125.1 (C-9b), 126.0 (C-14a), 134.5 (C-13a), 137.6 (C-14c), 142.5 (C-1), 143.4 (C-4a), 146.4 (C-3), 153.5 (C-6) ppm. IR (NaCl): v = 1467, 1572, $2926 \mathrm{~cm}^{-1}$. HRMS calcd. for $\mathrm{C}_{20} \mathrm{H}_{18} \mathrm{~N}_{3} \mathrm{~S}[\mathrm{M}+\mathrm{H}]^{+}$: 332.1216; found: 332.1214 .

\section{(3S,8aR)-3-(3-Indolylmethyl)-8a-methyl-5-oxo-2,3,6,7,8,8a- hexahydro-5H-oxazolo[3,2-a]pyridine (6):}

Method A: (S)-Tryptophanol (292 mg, $1.5 \mathrm{mmol})$ was added to a solution of 5-oxohexanoic acid (200 mg, $1.5 \mathrm{mmol})$ in toluene (23 $\mathrm{mL}$ ). The mixture was heated at reflux for $23 \mathrm{~h}$ with azeotropic elimination of water by a Dean-Stark apparatus. The mixture was cooled and concentrated. Flash chromatography of the residue (2:8 hexane/EtOAc) afforded a mixture of lactams 6 and 8a-epi-6 (340 $\mathrm{mg}, 80 \% ; 87: 13$ by GC-MS) as a light yellow foam and $50 \mathrm{mg}$ of 9 $(11 \%)$.

Method B: (S)-Tryptophanol (70 $\mathrm{mg}, 0.38 \mathrm{mmol})$ and 5oxohexanoic acid $(50 \mathrm{mg}, 0.38 \mathrm{mmol})$ in toluene $(3 \mathrm{~mL})$ were mixed in a capped $10 \mathrm{~mL}$ microwave vessel. The mixture was heated at $110{ }^{\circ} \mathrm{C}$ (average effective ramp time $=1 \mathrm{~min}$ ). The power was set at $100 \mathrm{~W}$ and the pressure was set at 15 bar for $10 \mathrm{~min}$. The solvent was evaporated, and the residue was dissolved in $\mathrm{CH}_{2} \mathrm{Cl}_{2}$. The organic phase was washed with $1 \mathrm{~N}$ aqueous $\mathrm{HCl}$ and saturated aqueous $\mathrm{NaHCO}_{3}$ solutions, dried, filtered, and evaporated to afford a mixture of 6 and 8a-epi-6 (83 mg, 77\%; 92:8 calculated by $\left.{ }^{1} \mathrm{H} \mathrm{NMR}\right)$.
Data for 6 (from the mixture): ${ }^{1} \mathrm{H} \mathrm{NMR}\left(400 \mathrm{MHz}, \mathrm{CDCl}_{3}, \mathrm{COSY}\right.$, $\left.\mathrm{HSQC}, 25^{\circ} \mathrm{C}\right): \delta=1.31\left(\mathrm{~s}, 3 \mathrm{H}, \mathrm{CH}_{3}\right), 1.56(\mathrm{td}, J=13.0,4.0 \mathrm{~Hz}, 1 \mathrm{H}$, H-8), 1.69-1.83 (m, 1H, H-7), 1.84-1.98 (m, 1H, H-7), 2.06 (dt, $J=$ 13.0, 3.6 Hz, 1H, H-8), 2.38 (ddd, $J=18.4,10.0,8.0 \mathrm{~Hz}, 1 \mathrm{H}, \mathrm{H}-6$ ), 2.54 (dd, $J=18.4,8.0 \mathrm{~Hz}, 1 \mathrm{H}, \mathrm{H}-6), 2.91$ (dd, $J=14.0,10.4 \mathrm{~Hz}$, $1 \mathrm{H}, \mathrm{CH}_{2}$ ), 3.55 (ddd, $J=14.0,3.2,0.8 \mathrm{~Hz}, 1 \mathrm{H}, \mathrm{CH}_{2}$ ), 3.85 (dd, $J=$ 8.5, 7.6 Hz, 1H, H-2), 3.98 (dd, $J=8.5,7.5 \mathrm{~Hz}, 1 \mathrm{H}, \mathrm{H}-2), 4.58$ (m, $1 \mathrm{H}, \mathrm{H}-3), 6.98$ (d, $\left.J=1.6 \mathrm{~Hz}, 1 \mathrm{H}, \mathrm{H}-2^{\prime}\right), 7.10(\mathrm{td}, J=8.0,1.2 \mathrm{~Hz}$, $1 \mathrm{H}, \mathrm{H}-5$ ') 7.17 (td, $J=7.6,1.2 \mathrm{~Hz}, 1 \mathrm{H}, \mathrm{H}-6$ ') 7.33 (d, $J=7.6 \mathrm{~Hz}$, 1H, H-7'), 7.77 (d, J = 8.0 Hz, 1H, H-4'), 8.61 (br. s, 1H, NH) ppm. ${ }^{13} \mathrm{C} \mathrm{NMR}\left(100.6 \mathrm{MHz}, \mathrm{CDCl}_{3}, 2{ }^{\circ} \mathrm{C}\right): \delta=16.9(\mathrm{C}-7), 23.7\left(\mathrm{CH}_{3}\right)$, $29.4\left(\mathrm{CH}_{2}\right), 30.4$ (C-6), 34.9 (C-8), 55.9 (C-3), 68.0 (C-2), 93.2 (C8a), 111.1 (C-7'), 111.5 (C-3'), 119.1 (C-4'), 119.3 (C-5'), 121.9 (C-6'), 122.2 (C-2'), 127.5 (C-3a'), 136.2 (C-7a'), 169.2 (CO) ppm. IR $(\mathrm{NaCl}): \mathrm{v}=1626,3284 \mathrm{~cm}^{-1}$. HRMS calcd. for $\mathrm{C}_{17} \mathrm{H}_{21} \mathrm{~N}_{2} \mathrm{O}_{2}[\mathrm{M}$ $+\mathrm{H}]^{+}:$285.1598; found: 285.1596 .

Data for 8a-epi-6 (from a enriched mixture): ${ }^{1} \mathrm{H}$ NMR (400 MHz, $\mathrm{CDCl}_{3}, 25^{\circ} \mathrm{C}$ ): $\delta=1.34$ (s, $\left.3 \mathrm{H}, \mathrm{CH}_{3}\right), 1.64(\mathrm{dd}, J=12.5,6.8 \mathrm{~Hz}$, $1 \mathrm{H}, \mathrm{H}-8$ ), 1.79-1.98 (m, 2H, H-7), 2.11 (ddd, $J=12.5,5.6,2.8 \mathrm{~Hz}$, $1 \mathrm{H}, \mathrm{H}-8$ ), 2.43-2.50 (m, 2H, H-6), 2.67 (dd, $J=13.8,10.0 \mathrm{~Hz}, 1 \mathrm{H}$, $\mathrm{CH}_{2}$ ), 3.78 (ddd, $J=13.8,2.9,0.8 \mathrm{~Hz}, 1 \mathrm{H}, \mathrm{CH}_{2}$ ), 3.95 (br. s, $1 \mathrm{H}$, H-2), 3.96 (br. s, 1H, H-2), 4.35 (m, 1H, H-3), 7.01 (d, $J=2.4 \mathrm{~Hz}$, $\left.1 \mathrm{H}, \mathrm{H}-2^{\prime}\right), 7.12(\mathrm{td}, J=8.0,1.2 \mathrm{~Hz}, 1 \mathrm{H}, \mathrm{H}-5$ ') 7.18 (td, $J=8.0,1.2$ $\mathrm{Hz}, 1 \mathrm{H}, \mathrm{H}-6$ ') 7.34 (d, $\left.J=7.6 \mathrm{~Hz}, 1 \mathrm{H}, \mathrm{H}-7^{\prime}\right), 7.81$ (d, $J=8.0 \mathrm{~Hz}$, $1 \mathrm{H}, \mathrm{H}-4$ '), 8.29 (br. s, $1 \mathrm{H}, \mathrm{NH}) \mathrm{ppm} .{ }^{13} \mathrm{C} \mathrm{NMR}(100.6 \mathrm{MHz}$, $\left.\mathrm{CDCl}_{3}, 25^{\circ} \mathrm{C}\right): \delta=16.7(\mathrm{C}-7), 22.9\left(\mathrm{CH}_{3}\right), 26.6\left(\mathrm{CH}_{2}\right), 29.6(\mathrm{C}-6)$, 34.6 (C-8), 56.3 (C-3), 67.3 (C-2), 93.2 (C-8a), 111.0 (C-7'), 112.5 (C-3'), 119.3 (C-4'), 119.5 (C-5'), 122.1 (C-6'), 122.3 (C-2'), 127.7 (C-3a'), 136.2 (C-7a'), 168.4 (CO) ppm. IR (NaCl): v = 1626, $3284 \mathrm{~cm}^{-1}$. HRMS calcd. for $\mathrm{C}_{17} \mathrm{H}_{21} \mathrm{~N}_{2} \mathrm{O}_{2}[\mathrm{M}+\mathrm{H}]^{+}:$: 285.1598; found: 285.1596 .

(4S,6S,12bS)-4,6-(Epoxymethano)-4-methyl-1,2,3,4,6,7,12,12boctahydroindolo[2,3-a]quinolizine (7): Lawesson's reagent (384 $\mathrm{mg}, 0.95 \mathrm{mmol}$ ) was added to a solution of lactams 6 and 8a-epi-6 (300 mg, $1.1 \mathrm{mmol})$ in DME $(10 \mathrm{~mL})$. The resulting mixture was heated at reflux for $4.5 \mathrm{~h}$, cooled, and concentrated to give a dark brown residue, which was dissolved in EtOAc. The solution was washed twice with saturated aqueous $\mathrm{NaHCO}_{3}$. The organic layer was dried, filtered, and concentrated to give $400 \mathrm{mg}$ of a yellow foam. Flash chromatography (hexane to $8: 2$ hexane/EtOAc) gave a mixture of thiolactams (179 mg, 54\%; 9:1 by GC-MS) as a white solid: ${ }^{1} \mathrm{H}$ NMR (400 MHz, $\mathrm{CDCl}_{3}, \mathrm{COSY}$, HSQC, $25{ }^{\circ} \mathrm{C}$, major epimer): $\delta=1.42$ (s, 3H, $\left.\mathrm{CH}_{3}\right), 1.67$ (dd, $\left.J=12.5,5.8 \mathrm{~Hz}, 1 \mathrm{H}, \mathrm{H}-8\right)$, 1.70-1.82 (m, 1H, H-7), 1.84-1.95 (m, 1H, H-7), 2.03-2.14 (m, 1H, H-8), 2.94 (dd, $J=13.7,10.7 \mathrm{~Hz}, 1 \mathrm{H}, \mathrm{H}-6$ ), 3.07-3.13 (m, 2H, H-6, $\mathrm{CH}_{2}$ ), 3.57-4.18 (m, 3H, $\left.\mathrm{CH}_{2}, \mathrm{H}-2\right), 4.92-5.19$ (m, 1H, H-3), 7.05 (d, $\left.J=1.9 \mathrm{~Hz}, 1 \mathrm{H}, \mathrm{H}-2^{\prime}\right), 7.12-7.18$ (m, 1H, H-5'), 7.19-7.25 (m, 1H, H-6'), 7.35 (d, $J=7.8 \mathrm{~Hz}, 1 \mathrm{H}, \mathrm{H}-7$ ') 7.99 (d, $J=7.8 \mathrm{~Hz}, 1 \mathrm{H}$, H-4'), 8.24 (br. s, $1 \mathrm{H}, \mathrm{NH}) \mathrm{ppm} .{ }^{13} \mathrm{C} \mathrm{NMR}\left(100.6 \mathrm{MHz}, \mathrm{CDCl}_{3}, 25\right.$ $\left.{ }^{\circ} \mathrm{C}\right): \delta=16.8(\mathrm{C}-7), 22.9\left(\mathrm{CH}_{3}\right), 27.7\left(\mathrm{CH}_{2}\right), 34.1(\mathrm{C}-6), 39.6(\mathrm{C}-8)$, 61.1 (C-3), 67.1 (C-2), 94.6 (C-8a), 111.0 (C-7'), 111.3 (C-3'), 119.5 (C-4'), 119.6 (C-5'), 122.1 (C-6'), 122.3 (C-2'), 127.4 (C3a'), 136.1 (C-7a'), 197.0 (CO) ppm. BnBr (134 $\mu \mathrm{L}, 1.13 \mathrm{mmol})$ was added to a solution, kept in the dark, of the above thiolactams (170 mg, $0.57 \mathrm{mmol}$ ) in $\mathrm{CH}_{3} \mathrm{CN}(4.5 \mathrm{~mL}$ ), and the resulting mixture was heated at $60{ }^{\circ} \mathrm{C}$ for $48 \mathrm{~h}$. The solvent was removed under reduced pressure, and the dark yellow residue was dissolved in $\mathrm{MeOH}(15 \mathrm{~mL})$. Then, $\mathrm{NaBH}_{4}(65 \mathrm{mg}, 1.71 \mathrm{mmol})$ was added at 
$-78{ }^{\circ} \mathrm{C}$, and the mixture was allowed to reach room temperature and stirred for $4.5 \mathrm{~h}$. Acetone $(0.5 \mathrm{~mL})$ was added, and the solvent was evaporated. The resulting residue was dissolved in $\mathrm{CH}_{2} \mathrm{Cl}_{2}$, and the solution was washed with brine. The organic phase was dried and concentrated to give a yellow oil $(300 \mathrm{mg})$, which was chromatographed (7:3 hexane/EtOAc) to afford 7 (78 $\mathrm{mg}, 51 \%)$ as a yellow oil: $[\alpha]^{22}{ }_{\mathrm{D}}=-109.1\left(c=0.7, \mathrm{CHCl}_{3}\right) .{ }^{1} \mathrm{H} \mathrm{NMR}(400 \mathrm{MHz}$, $\mathrm{CDCl}_{3}, \mathrm{COSY}$, HSQC, $\left.25^{\circ} \mathrm{C}\right): \delta=1.20\left(\mathrm{~s}, 3 \mathrm{H}, \mathrm{CH}_{3}\right), 1.46(\mathrm{~m}, 1 \mathrm{H}$, $\mathrm{H}-2), 1.62$ (m, 2H, H-1, H-3), 1.93 (m, 2H, H-1, H-3), 2.05 (m, 1H, H-2), 2.58 (ddd, $J=14.4,10.0,2.4 \mathrm{~Hz}, 1 \mathrm{H}, \mathrm{H}-7$ ), 3.01 (ddd, $J=$ 14.4, 4.4, $1.6 \mathrm{~Hz}, 1 \mathrm{H}, \mathrm{H}-7), 3.32$ (m, 1H, H-6), 3.72 (t, $J=7.5 \mathrm{~Hz}$, $1 \mathrm{H}, \mathrm{CH}_{2} \mathrm{O}$ ), 3.84 (br. dd, $J=10.8,1.6 \mathrm{~Hz}, 1 \mathrm{H}, \mathrm{H}-12 \mathrm{~b}$ ), 4.23 (t, $J=$ $7.5 \mathrm{~Hz}, 1 \mathrm{H}, \mathrm{CH}_{2} \mathrm{O}$ ), 7.09 (dd, $\left.J=7.2,1.2 \mathrm{~Hz}, 1 \mathrm{H}, \mathrm{H}-9\right), 7.14$ (dd, $J$ $=7.2,1.2 \mathrm{~Hz}, 1 \mathrm{H}, \mathrm{H}-10), 7.31(\mathrm{~d}, J=7.2 \mathrm{~Hz}, 1 \mathrm{H}, \mathrm{H}-11), 7.47$ (d, $J$ $=7.2 \mathrm{~Hz}, 1 \mathrm{H}, \mathrm{H}-8$ ), 7.88 (br. s, 1H, NH) ppm. NOESY 1D: positive NOE effects: 4-Me/6-H, 4-Me/12b-H, and 6-H/12b-H. ${ }^{13} \mathrm{C}$ $\operatorname{NMR}\left(100.6 \mathrm{MHz}, \mathrm{CDCl}_{3}, 25^{\circ} \mathrm{C}\right): \delta=13.9\left(\mathrm{CH}_{3}\right), 21.9(\mathrm{C}-2), 26.2$ (C-7), 30.8 (C-1), 36.6 (C-3), 51.3 (C-12b), 52.5 (C-6), 70.3 $\left(\mathrm{CH}_{2} \mathrm{O}\right), 91.5(\mathrm{C}-4), 107.7$ (C-7a), 110.7 (C-11), 118.0 (C-8), 119.4 (C-9), 121.4 (C-10), 127.2 (C-7b), 135.5 (C-12a), 136.5 (C-11a) ppm. IR (NaCl): $v=3267 \mathrm{~cm}^{-1}$. HRMS calcd. for $\mathrm{C}_{17} \mathrm{H}_{21} \mathrm{~N}_{2} \mathrm{O}[\mathrm{M}+$ $\mathrm{H}^{+}$: 269.1648; found: 269.1644 .

Treatment of 6 under Bischler-Napieralski conditions: $\mathrm{POCl}_{3}$ $(500 \mu \mathrm{L}, 5.5 \mathrm{mmol})$ was added to a solution of lactams $\mathbf{6}$ and epi-6 $(100 \mathrm{mg}, 0.35 \mathrm{mmol})$ in toluene $(5 \mathrm{~mL})$, and the mixture was heated at $100{ }^{\circ} \mathrm{C}$ for $2 \mathrm{~h}$. After cooling, the solvent was removed, and the brown residue was dissolved in anhydrous $\mathrm{MeOH}(7 \mathrm{~mL})$. Then, $\mathrm{NaBH}_{4}$ (excess, until basic $\mathrm{pH}$ ) was slowly added to the solution at $0{ }^{\circ} \mathrm{C}$, and the mixture was allowed to reach room temperature and stirred for $3 \mathrm{~h}$. After cooling at $0{ }^{\circ} \mathrm{C}$, the mixture was quenched with $\mathrm{H}_{2} \mathrm{O}$ and extracted with EtOAc. The organic layer was washed with brine, dried, and concentrated to give an oil (114 mg). Flash chromatography (8:2 hexane/EtOAc to $95: 5$ EtOAc/MeOH) gave pentacycle $7(31 \mathrm{mg}, 33 \%)$, tetracycle 9 (6 $\mathrm{mg}, 6 \%$ ) and tetracycle 8 (14 mg, 19\%).

$\mathrm{NaBH}_{4}$ reduction of 7: $\mathrm{NaBH}_{4}(85 \mathrm{mg}, 2.25 \mathrm{mmol})$ was slowly added $\left(0{ }^{\circ} \mathrm{C}\right)$ to a solution of $7(120 \mathrm{mg}, 0.45 \mathrm{mmol})$ in $\mathrm{MeOH}(10$ $\mathrm{mL}$ ), and the suspension was stirred at room temperature for $1 \mathrm{~h}$. The mixture was cooled at $0{ }^{\circ} \mathrm{C}$, quenched with $\mathrm{H}_{2} \mathrm{O}$, and extracted with $\mathrm{CH}_{2} \mathrm{Cl}_{2}$. The combined organic extracts were washed with brine, dried, and concentrated to give a yellow oil which was chromatographed (EtOAc) affording tetracycle 8 (108 mg, 90\%).

Data for 8: Light yellow oil. $[\alpha]^{22}{ }_{\mathrm{D}}=+36.5\left(c=1.1, \mathrm{CHCl}_{3}\right) .{ }^{1} \mathrm{H}$ NMR (400 MHz, CDCl 3 , COSY, HSQC, $\left.25^{\circ} \mathrm{C}\right): \delta=1.21(\mathrm{~d}, J=$ $6.8 \mathrm{~Hz}, 3 \mathrm{H}, \mathrm{CH}_{3}$ ), 1.30-1.39 (m, 1H, H-3), 1.40-1.52 (m, 1H, H-2), 1.56-1.67 (m, 2H, H-1, H-3), 1.81-1.97 (m, 2H, H-1, H-2), 2.76 (dd, $J=15.0,5.2 \mathrm{~Hz}, 1 \mathrm{H}, \mathrm{H}-7), 2.96(\mathrm{dd}, J=15.0,3.1 \mathrm{~Hz}, 1 \mathrm{H}, \mathrm{H}-7)$, 3.14-3.24 (m, 1H, H-4), 3.49 (dd, $J=9.6,8.4 \mathrm{~Hz}, 1 \mathrm{H}, \mathrm{CH}_{2} \mathrm{O}$ ), 3.53-3.64 (m, 2H, H-12b, $\mathrm{CH}_{2} \mathrm{O}$ ), 4.01 (br. d, $J=9.2 \mathrm{~Hz}, 1 \mathrm{H}, \mathrm{H}-6$ ), $7.08(\mathrm{td}, J=7.6,1.2 \mathrm{~Hz}, 1 \mathrm{H}, \mathrm{H}-10), 7.13(\mathrm{td}, J=7.6,1.2 \mathrm{~Hz}, 1 \mathrm{H}$, H-9), 7.30 (d, $J=7.6 \mathrm{~Hz}, 1 \mathrm{H}, \mathrm{H}-8), 7.47$ (d, $J=7.1 \mathrm{~Hz}, 1 \mathrm{H}, \mathrm{H}-11$ ), 7.80 (br. s, 1H, NH) ppm. NOESY 1D: positive NOE effect: 4$\mathrm{H} / 6-\mathrm{H} .{ }^{13} \mathrm{C} \mathrm{NMR}\left(100.6 \mathrm{MHz}, \mathrm{CDCl}_{3}, 25{ }^{\circ} \mathrm{C}\right): \delta=20.0\left(\mathrm{CH}_{3}\right), 23.2$ (C-7), 23.3 (C-1), 30.3 (C-2), 33.8 (C-3), 54.5 (C-4), 54.9 (C-12b), 54.7 (C-6), $64.2\left(\mathrm{CH}_{2} \mathrm{O}\right), 107.1(\mathrm{C}-7 \mathrm{a}), 110.0(\mathrm{C}-8), 118.1(\mathrm{C}-11)$, 119.3 (C-10), 121.2 (C-9), 127.7 (C-7b), 135.5 (C-12a), 135.9 (C- 11a) ppm. IR $(\mathrm{NaCl}): v=1453,3257 \mathrm{~cm}^{-1}$. HRMS calcd. for $\mathrm{C}_{17} \mathrm{H}_{22} \mathrm{~N}_{2} \mathrm{O}[\mathrm{M}+\mathrm{H}]^{+}: 271.1805$; found: 271.1807 .

\section{(6S,12bR)-6-(Hydroxymethyl)-12b-methyl-4-oxo-}

1,2,3,4,6,7,12,12b-octahydroindolo[2,3-a]quinolizine (9): TFA (0.9 $\mathrm{M}$ solution in $\mathrm{CH}_{2} \mathrm{Cl}_{2}, 4 \mathrm{~mL}$ ) was added to a solution of lactams 6 and epi-6 (100 mg, $0.35 \mathrm{mmol})$ in $\mathrm{CH}_{2} \mathrm{Cl}_{2}(2 \mathrm{~mL})$, and the resulting mixture was stirred at room temperature for $20 \mathrm{~h}$. The solvent was removed under reduced pressure, and the residue was dissolved in $\mathrm{CH}_{2} \mathrm{Cl}_{2}(6 \mathrm{~mL})$. Then, $\mathrm{KOH}(150 \mathrm{mg}, 2.7 \mathrm{mmol})$ was added, and the suspension was stirred at room temperature for $20 \mathrm{~h}$. The resulting mixture was diluted with $\mathrm{CH}_{2} \mathrm{Cl}_{2}$ and washed twice with water. The organic layer was dried, filtered, and concentrated. Flash chromatography (8:2 hexane/EtOAc to EtOAc) gave tetracycle 9 (66 mg, 66\%) and 12b-epi-9 (20 mg, 20\%).

Data for 9: Brown solid. $[\alpha]^{22}{ }_{\mathrm{D}}=+132.6(c=0.9, \mathrm{MeOH}) .{ }^{1} \mathrm{H}$ NMR $\left(\mathrm{CD}_{3} \mathrm{OD}, 400 \mathrm{MHz}, \mathrm{COSY}, \mathrm{HSQC}, 25^{\circ} \mathrm{C}\right): \delta=1.67(\mathrm{~s}, 3 \mathrm{H}$, $\left.\mathrm{CH}_{3}\right), 1.80$ (td, $\left.J=13.6,4.6 \mathrm{~Hz}, 1 \mathrm{H}, \mathrm{H}-1\right), 1.99-1.85$ (m, $\left.1 \mathrm{H}, \mathrm{H}-2\right)$, 2.25-2.05 (m, 1H, H-2), 2.35 (dt, $J=13.3,3.2 \mathrm{~Hz}, 1 \mathrm{H}, \mathrm{H}-1), 2.54$ (ddd, $J=18.5,9.7,9.0 \mathrm{~Hz}, 1 \mathrm{H}, \mathrm{H}-3), 2.67$ (dd, $J=18.5,7.5 \mathrm{~Hz}$, $1 \mathrm{H}, \mathrm{H}-3$ ), 2.81 (dd, $J=15.8,6.2 \mathrm{~Hz}, 1 \mathrm{H}, \mathrm{H}-7), 3.04$ (dd, $J=15.8$, $1.1 \mathrm{~Hz}, 1 \mathrm{H}, \mathrm{H}-7), 3.54$ (dd, $J=10.3,6.1 \mathrm{~Hz}, 1 \mathrm{H}, \mathrm{CH}_{2} \mathrm{O}$ ), 3.62 (dd, $\left.J=10.3,8.9 \mathrm{~Hz}, 1 \mathrm{H}, \mathrm{CH}_{2} \mathrm{O}\right), 5.45(\mathrm{dd}, J=8.9,6.1 \mathrm{~Hz}, 1 \mathrm{H}, \mathrm{H}-6)$, 7.03 (ddd, $J=8.0,7.1,1.0 \mathrm{~Hz}, 1 \mathrm{H}, \mathrm{H}-10), 7.11$ (ddd, $J=8.2,7.1$, $1.2 \mathrm{~Hz}, 1 \mathrm{H}, \mathrm{H}-9), 7.33$ (d, $J=8.1 \mathrm{~Hz}, 1 \mathrm{H}, \mathrm{H}-8), 7.46$ (d, $J=7.6 \mathrm{~Hz}$, $1 \mathrm{H}, \mathrm{H}-11)$ ppm. ${ }^{13} \mathrm{C}$ NMR $\left(\mathrm{CD}_{3} \mathrm{OD}, 100.6 \mathrm{MHz}, 25{ }^{\circ} \mathrm{C}\right): \delta=16.8$ (C-2), 21.9 (C-7), $27.6\left(\mathrm{CH}_{3}\right), 31.4$ (C-3), 38.2 (C-1), 50.7 (C-6), 57.3 (C-12b), $63.8\left(\mathrm{CH}_{2} \mathrm{O}\right), 104.4(\mathrm{C}-7 \mathrm{a}), 111.9$ (C-11), 118.9 (C8), 119.9 (C-10), 122.5 (C-9), 128.1 (C-7b), 137.9 (C-12a), 138.3 (C-11a), 173.1 (CO) ppm. IR (NaCl): v = 1609, $3268 \mathrm{~cm}^{-1}$. HRMS calcd. for $\mathrm{C}_{17} \mathrm{H}_{21} \mathrm{~N}_{2} \mathrm{O}_{2}[\mathrm{M}+\mathrm{H}]^{+}$: 285.1598; found: 285.1593 .

Data for 12b-epi-9: $[\alpha]^{22}{ }_{D}=-168.8(c=1.0, \mathrm{MeOH}) .{ }^{1} \mathrm{H}$ NMR (400 MHz, $\left.\mathrm{CD}_{3} \mathrm{OD}, 25^{\circ} \mathrm{C}\right): \delta=1.74\left(\mathrm{~s}, 3 \mathrm{H}, \mathrm{CH}_{3}\right), 1.75-1.84(\mathrm{~m}$, $1 \mathrm{H}, \mathrm{H}-2), 1.88-2.06$ (m, 1H, H-1, H-2), 2.30-2.47 (m, 2H, H-1, H3), 2.52-2.62 (m, 1H, H-3), 2.84 (dd, $J=15.0,3.1 \mathrm{~Hz}, 1 \mathrm{H}, \mathrm{H}-7)$, 3.07 (dd, $J=15.0,8.7 \mathrm{~Hz}, 1 \mathrm{H}, \mathrm{H}-7), 3.90-4.05$ (m, 3H, H-6, $\mathrm{CH}_{2} \mathrm{O}$ ), 7.02 (ddd, $J=8.0,7.1,1.2 \mathrm{~Hz}, 1 \mathrm{H}, \mathrm{H}-10$ ), 7.09 (ddd, $J=$ 8.0, 7.1, 1.2 Hz, 1H, H-9), 7.32 (d, $J=8.0 \mathrm{~Hz}, 1 \mathrm{H}, \mathrm{H}-8), 7.44$ (d, $J$ $=7.8 \mathrm{~Hz}, 1 \mathrm{H}, \mathrm{H}-11)$ ppm. ${ }^{13} \mathrm{C} \mathrm{NMR}\left(100.6 \mathrm{MHz}, \mathrm{CD}_{3} \mathrm{OD}, 25{ }^{\circ} \mathrm{C}\right)$ : $\delta=17.6(\mathrm{C}-2), 23.7(\mathrm{C}-7), 27.2\left(\mathrm{CH}_{3}\right), 33.9(\mathrm{C}-3), 36.5(\mathrm{C}-1), 59.4$ (C-6), 61.1 (C-12b), $64.3\left(\mathrm{CH}_{2} \mathrm{O}\right), 108.5$ (C-7a), 112.1 (C-11), 118.8 (C-8), 120.0 (C-10), 122.4 (C-9), 127.9 (C-7b), 137.9 (C12a), 140.1 (C-11a), 174.4 (CO) ppm. IR (NaCl): v = 1613, 3268 $\mathrm{cm}^{-1}$. HRMS calcd. for $\mathrm{C}_{17} \mathrm{H}_{21} \mathrm{~N}_{2} \mathrm{O}_{2}[\mathrm{M}+\mathrm{H}]^{+}:$285.1598; found: 285.1597 .

Data for 10: $[\alpha]^{22}{ }_{\mathrm{D}}=+160.9\left(c=1.0, \mathrm{CHCl}_{3}\right) .{ }^{1} \mathrm{H} \mathrm{NMR}(400 \mathrm{MHz}$, $\mathrm{CDCl}_{3}, \mathrm{COSY}$, HSQC, $\left.25^{\circ} \mathrm{C}\right): \delta=1.71\left(\mathrm{~s}, 3 \mathrm{H}, \mathrm{CH}_{3}\right), 1.85$ (td, $J=$ 13.0, 4.6 Hz, 1H, H-1), 1.90-1.99 (m, 1H, H-2), 2.03-2.21 (m, $1 \mathrm{H}$, H-2), 2.27 (d, $J=13.0 \mathrm{~Hz}, 1 \mathrm{H}, \mathrm{H}-1), 2.61$ (dt, $J=18.5,9.5 \mathrm{~Hz}, 1 \mathrm{H}$, H-3), 2.74 (dd, $J=18.5,7.5 \mathrm{~Hz}, 1 \mathrm{H}, \mathrm{H}-3$ ), 2.95 (dd, $J=16.0,6.2$ $\mathrm{Hz}, 1 \mathrm{H}, \mathrm{H}-7), 3.10$ (dd, $J=16.0,1.0 \mathrm{~Hz}, 1 \mathrm{H}, \mathrm{H}-7), 3.55$ (dd, $J=$ $\left.10.5,6.7 \mathrm{~Hz}, 1 \mathrm{H}, \mathrm{CH}_{2} \mathrm{Cl}\right), 3.62\left(\mathrm{dd}, J=10.5,8.7 \mathrm{~Hz}, 1 \mathrm{H}, \mathrm{CH}_{2} \mathrm{Cl}\right)$, $5.72(\mathrm{dd}, J=14.1,6.8 \mathrm{~Hz}, 1 \mathrm{H}, \mathrm{H}-6), 7.11-7.17$ (m, 1H, H-10), 7.17-7.23 (m, 1H, H-9), 7.36 (d, $J=8.0 \mathrm{~Hz}, 1 \mathrm{H}, \mathrm{H}-8), 7.53$ (d, $J=$ $7.8 \mathrm{~Hz}, 1 \mathrm{H}, \mathrm{H}-11), 8.86$ (br. s, $1 \mathrm{H}, \mathrm{NH}) \mathrm{ppm} .{ }^{13} \mathrm{C}$ NMR $(100.6$ $\left.\mathrm{MHz}, \mathrm{CDCl}_{3}, 25^{\circ} \mathrm{C}\right): \delta=16.0(\mathrm{C}-2), 22.2(\mathrm{C}-7), 27.7\left(\mathrm{CH}_{3}\right), 30.7$ (C-3), 37.7 (C-1), 45.5 (C-6), $48.7\left(\mathrm{CH}_{2} \mathrm{Cl}\right), 55.7$ (C-12b), 104.1 
(C-7a), 111.0 (C-11), 118.4 (C-8), 119.6 (C-10), 122.2 (C-9), 126.8 (C-7b), 136.2 (C-12a), 136.6 (C-11a), 170.7 (CO) ppm. IR ( NaCl): $v=1397,1611,3268 \mathrm{~cm}^{-1}$. HRMS calcd. for $\mathrm{C}_{17} \mathrm{H}_{20} \mathrm{ClN}_{2} \mathrm{O}_{2}[\mathrm{M}+$ $\mathrm{H}^{+}$: 303.1259; found: 303.1251 .

\section{Acknowledgments}

Financial support from the Spanish Ministries of Science and Innovation (Project CTQ2009-07021/BQU) and Economy and Competitiveness (Project CTQ2012-35250), and the AGAUR, Generalitat de Catalunya (Grant 2009-SGR-1111) is gratefully acknowledged.

[1] For reviews, see: a) M. Amat, M. Pérez, J. Bosch, Synlett 2011, 143-160; b) M. M. M. Santos in: Heterocyclic Targets in Advanced Organic Synthesis (Eds.: M. do C. Carreiras, J. Marco-Contelles), Research Signpost, Kerala, India, 2011, pp. 69-82. See also: c) S. M Allin, C. I. Thomas, K. Doyle, M. R. J. Elsegood, J. Org. Chem. 2005, 70, 357-359; d) S. M. Allin, J. S. Khera, J. Witherington, M R. J. Elsegood, Tetrahedron Lett. 2006, 47, 5737-5739; e) M. Amat, A. Gómez-Esqué, C. Escolano, M. M. M. Santos, E. Molins, J. Bosch, J. Org. Chem. 2009, 74, 1205-1211.

[2] a) S. M. Allin, C. I. Thomas, J. E. Allard, K. Doyle, M. R. J. Elsegood, Tetrahedron Lett. 2004, 45, 7103-7105; b) O. Bassas, N. Llor, M. M. M. Santos, R. Griera, E. Molins, M. Amat, J. Bosch, Org. Lett. 2005, 7, 2817-2820; c) M. Amat, M. M. M. Santos, O. Bassas, N. Llor, C. Escolano, A. Gómez-Esqué, E. Molins, S. M. Allin, V. McKee, J. Bosch, J. Org. Chem. 2007, 72, 5193-5201; d) S M. Allin, L. J. Duffy, P. C. Bulman Page, V. McKee, M. J. McKenzie, Tetrahedron Lett. 2007, 48, 4711-4714; e) S. M. Allin, L. J. Duffy, J. M. R. Towler, P. C. Bulman Page, M. R. J. Elsegood, B. Saha, Tetrahedron 2009, 65, 10230-10234.

[3] M. Amat, L. Navío, N. Llor, E. Molins, J. Bosch, Org. Lett. 2012, 14, 210-213.

[4] M. Amat, M. M. M. Santos, A. M. Gómez, D. Jokic, E. Molins, J. Bosch, Org. Lett. 2007, 9, 2907-2910.

[5] M. Pérez, F. Arioli, G. Rigacci, M. M. M. Santos, A. Gómez-Esqué, C. Escolano, P. Florindo, C. Ramos, J. Bosch, M. Amat, Eur. J. Org. Chem. 2011, 3858-3863.

[6] a) Isolation: G. Massiot, P. Thépenier, M.-J. Jacquier, L. Le MenOlivier, R. Verpoorte, C. Delaude, Phytochemistry 1987, 26 , 2839-2846; b) Absolute configuration: D. Arbain, D. P. Putra, M. V. Sargent, Aust. J. Chem. 1993, 46, 977-985; c) First enantioselective synthesis: M. Ohba, H. Kubo, T. Fujii, H. Ishibashi, M. V. Sargent, D. Arbain, Tetrahedron Lett. 1997, 38, 6697-6700.

[7] For precedents, see ref. ${ }^{[1 \mathrm{c}-\mathrm{e}, 2 \mathrm{a}, 2 \mathrm{c}, 4]}$

[8] Minor amounts of 10b-epi-2 were also detected (ratio 2:10b-epi-2 96:4). Cyclocondensation reactions of phenylglycinol with $\delta$-keto esters lead to oxazolopiperidone lactams with a trans-relationship between the methine proton and the angular substituent of the oxazolidine ring: M. Amat, O. Bassas, N. Llor, M. Cantó, M. Pérez, E. Molins, J. Bosch, Chem. Eur. J. 2006, 12, 7872-7881

[9] For reviews on the chemistry and cyclizations of $N$-acyl iminium ions, see: a) W. N. Speckamp, M. J. Moolenaar, Tetrahedron 2000, 56, 3817-3856; b) B. E. Maryanoff, H.-C. Zhang, J. H. Cohen, I. J. Turchi, C. A. Maryanoff, Chem. Rev. 2004, 104, 1431-1628.

[10] For the generation of related oxazolinium salts by treatment of aminoalcohol-derived lactams with $\mathrm{POCl}_{3}$ or $\mathrm{POBr}_{3}$, see: a) A Castro, J. Ramírez, J. Juárez, J. L. Terán, L. Orea, A. Galindo, D. Gnecco, Heterocycles 2007, 71, 2699-2708; b) D. Gnecco, A. M Lumbreras, J. L. Terán, A. Galindo, J. R. Juárez, M. L. Orea, A
Castro, R. G. Enríquez, W. F. Reynolds, Heterocycles 2009, 78, 2589-2594; c) see also ref. ${ }^{[5]}$

[11] For reviews, see: a) M. Lounasmaa, M. Berner, A. Tolvanen, Heterocycles 1998, 48, 1275-1290; b) M. Lounasmaa, M. Berner, M. Brunner, H. Suomalainen, A. Tolvanen, Tetrahedron 1998, 54, 10205-10216.

[12] The kinetically controlled product in $\alpha$-amidoalkylation reactions from tryptophanol-derived lactams possesses a trans relative stereochemistry between the methine proton of the tryptophanol moiety and the angular substituent at $\mathrm{C}-12 \mathrm{~b}$ (indolo[2,3a]quinolizidine numbering): see ref. ${ }^{[1 \mathrm{c}, \mathrm{d}, 2,5]}$

[13] P. Deslongchamps, Stereoelectronic Effects in Organic Chemistry; Pergamon Press, Oxford, 1983, p. 221.

[14] For precedents for the direct conversion of alcohols into thiols involving the nucleophilic attack of the alcohol to the monomeric species of Lawesson's reagent present in solution to form an $O$-alkyl phosphonodithioic acid intermediate, see: T. Nishio, J. Chem. Soc., Perkin Trans I 1993, 1113-1117.

[15] Minor amounts $(\sim 10 \%)$ of indoloquinolizidine 9 were also formed. A similar cyclocondensation from 4-oxopentanoic acid has been reported to give the corresponding indoloindolizidine as the main product by a mechanism that involves an initial Pictet-Spengler cyclization leading to a tetrahydro- $\beta$-carboline derivative, which undergoes a final lactamization: S. M. Allin, C. I. Thomas, J. E. Allard, M. Duncton, M. R. J. Elsegood, M. Edgar, Tetrahedron Lett. 2003, 44, 2335-2337.

[16] For the generation of aminoalcohol-derived oxazolopiperidone lactams using the microwave-assisted procedure, see: M. Jida, R. Deprez-Poulain, S. Malaquin, P. Roussel, F. Agbossou-Niedercorn, B. Deprez, G. Laconde, Green Chem. 2010, 12, 961-964.

[17] Minor amounts $(\sim 5 \%)$ of the $\alpha$-amidoalkylation product 10, in which the substitution of the hydroxyl group by chloride has also occurred, were detected from the crude mixture.

[18] Minor amounts of the epimer 4-epi-8 were detected by GC-MS (93:7 ratio).

[19] CCDC-909407 (for 9) and CCDC-909408 (for 12b-epi-9) contain the supplementary crystallographic data for this paper. These data can be obtained free of charge from The Cambridge Crystallographic Data Centre via www.ccdc.cam.ac.uk/data_request/cif.

[20] For related cyclizations where a substituent $\alpha$ to the amide nitrogen acts as an element of stereocontrol, see: a) B. E. Maryanoff, D. F. McComsey, H. R. Almond Jr., M. S. Mutter, G. W. Bemis, R. R. Whittle, R. A. Olofson, J. Org. Chem. 1986, 51, 1341-1346; b) R. H. Huizenga, U. K. Pandit, Tetrahedron 1992, 48, 6521-6528; c) S. M. Allin, S. L. James, W. P. Martin, T. A. D. Smith, M. R. J. Elsegood, J. Chem. Soc., Perkin Trans I 2001, 3029-3036; d) A. Ardeo, E. García, S. Arrasate, E. Lete, N. Sotomayor, Tetrahedron Lett. 2003, 44, 8445-8448; e) T. E. Nielsen, M. Meldal, J. Org. Chem. 2004, 69, 3765-3773; f) E. García, S. Arrasate, E. Lete, N. Sotomayor, J. Org. Chem. 2005, 70, 10368-10374. See also ref. ${ }^{[1,2]}$

[21] F. Bracher, K. Mink, Liebigs Ann. 1995, 645-647.

Received: ((will be filled in by the editorial staff)) Published online: ((will be filled in by the editorial staff)) 


\section{Entry for the Table of Contents}

\section{Layout 2:}
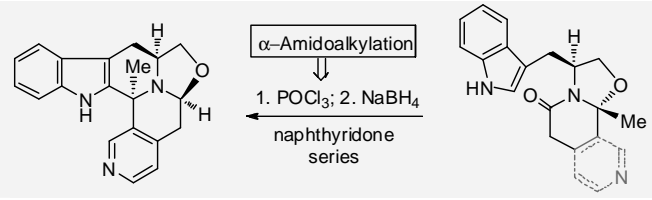

Cyclization on the lactam carbonyl 1. $P$ $\underset{\text { piperidone }}{\longrightarrow}$ series

Cyclizations on the lactam carbonyl from tryptophanol-derived oxazolopiperidone lactams are studied. A pyridine ring fused to the piperidone moiety exerts a dramatic effect on the regioselectivity of the cyclization, exclusively leading to $\alpha$-amidoalkylation products.
M. Amat, N. Llor, F. Subrizi, M. Pérez, E. Molins, and J. Bosch Page No. - Page No.

Studies on the Regioselectivity of the Cyclization of Tryptophanol-Derived Oxazolopiperidone Lactams.

Keywords: Lactams / Asymmetric synthesis / Cyclization /BischlerNapieralski / $\alpha$-Amidoalkylation 


\section{Supporting Information}

NMR-H $\mathrm{H}^{1 / \text { Mercury- }} 400$

$\mathrm{T}=25^{\circ} \mathrm{C} / \mathrm{CDCl}_{3}$

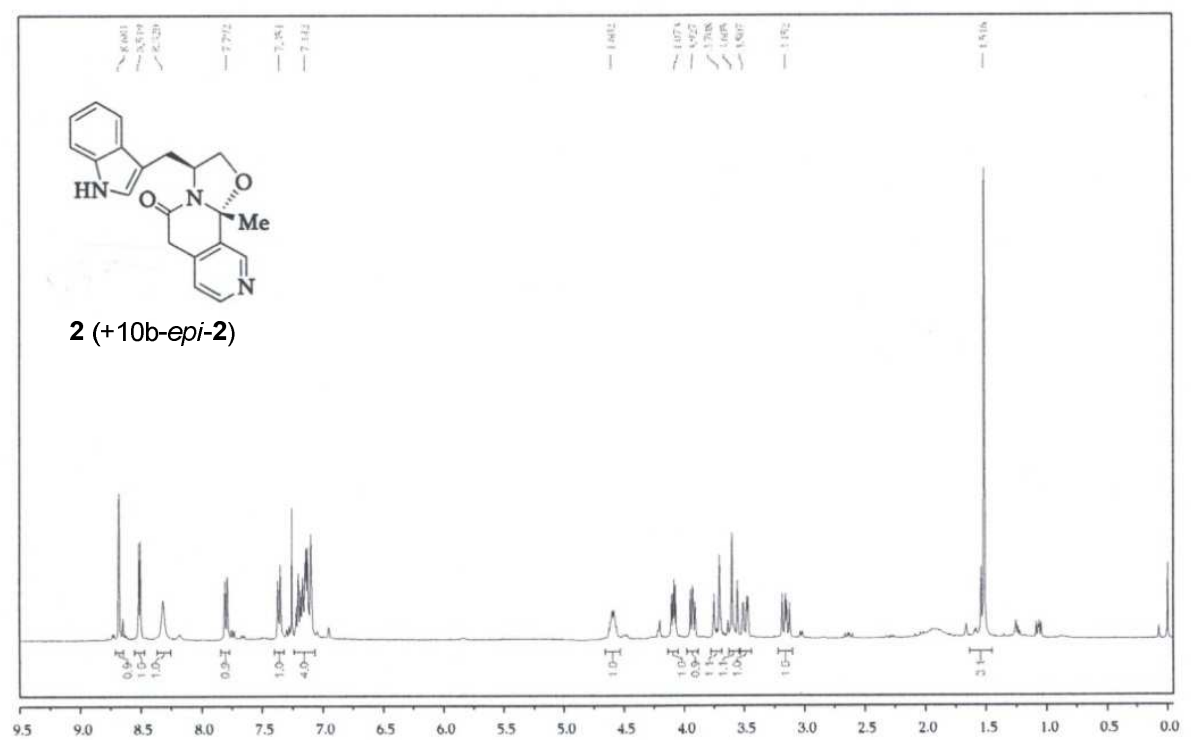

NMR-C $13 /$ Mercury- 400

$\mathrm{T}=25^{\circ} \mathrm{C} / \mathrm{CDCl}_{3}$

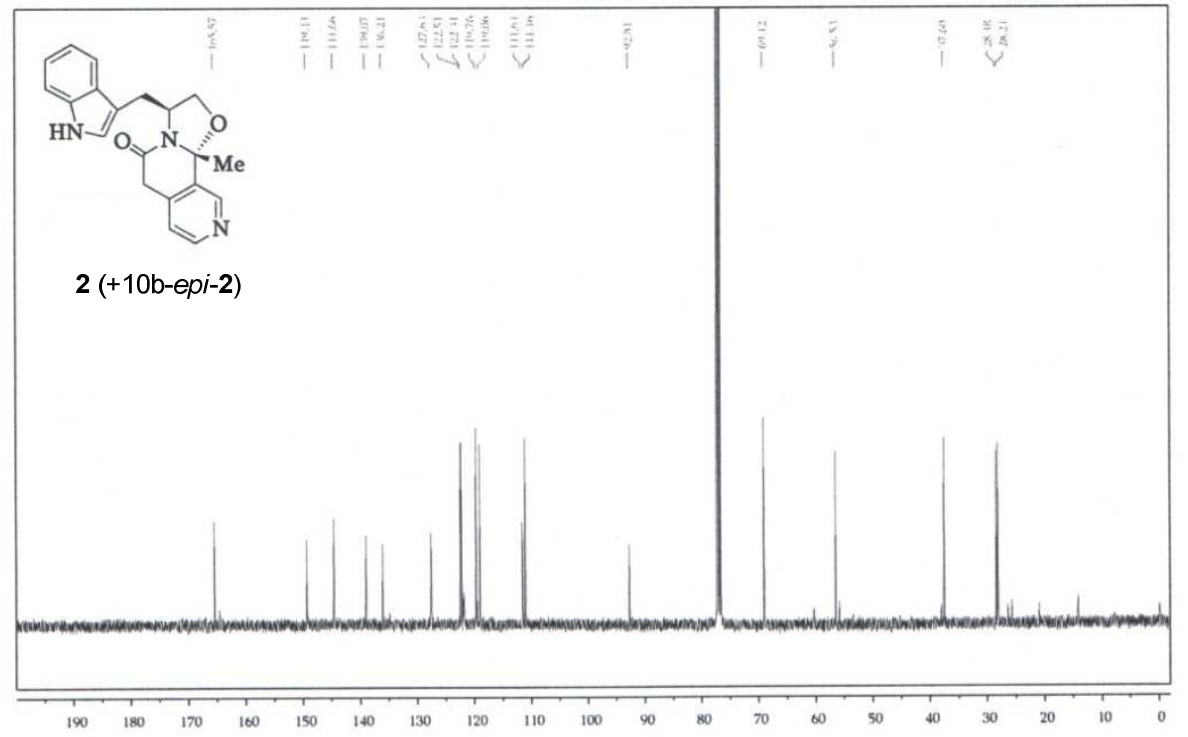


NMR-H ${ }^{1} /$ Mercury-400

$\mathrm{T}=25^{\circ} \mathrm{C} / \mathrm{CDCl}_{3}$

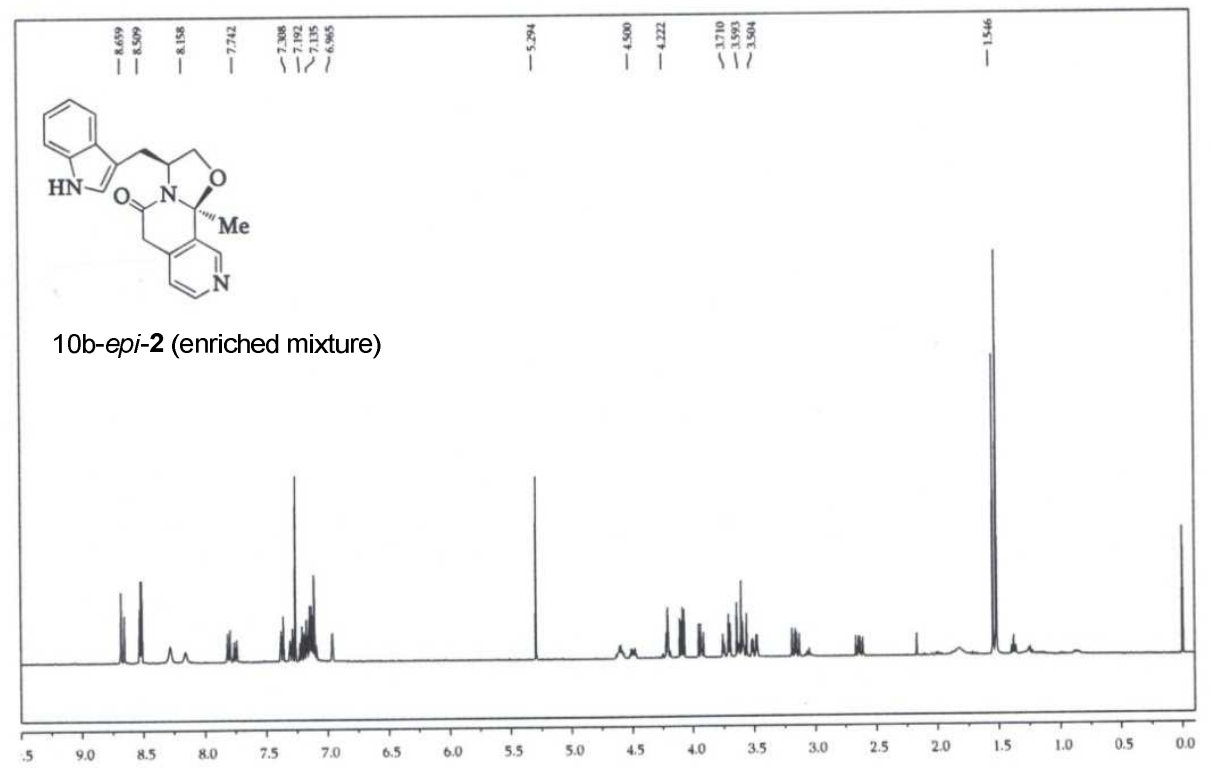


NMR-H ${ }^{1} /$ Mercury-400

$\mathrm{T}=25^{\circ} \mathrm{C} / \mathrm{CDCl}_{3}$

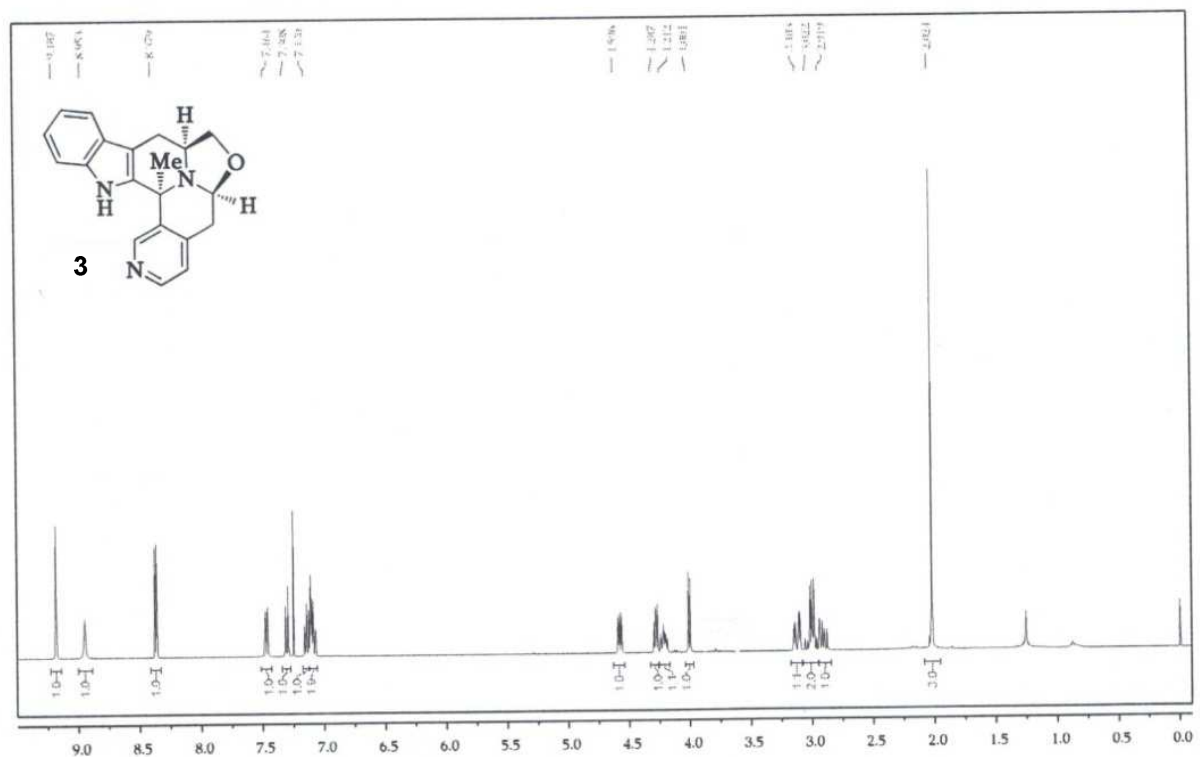

NMR-C ${ }^{13} /$ Mercury-400

$\mathrm{T}=25^{\circ} \mathrm{C} / \mathrm{CDCl}_{3}$

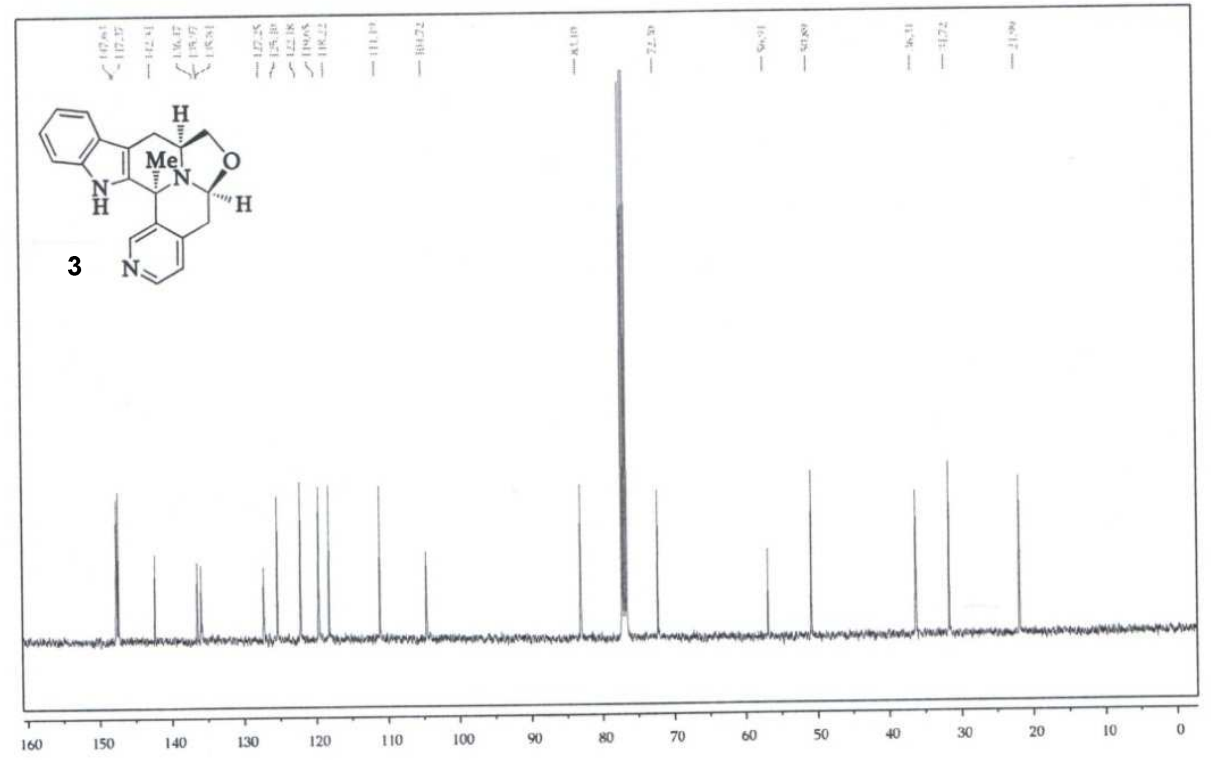


NMR-H ${ }^{1} /$ Mercury-400

$\mathrm{T}=25^{\circ} \mathrm{C} / \mathrm{CDCl}_{3}$

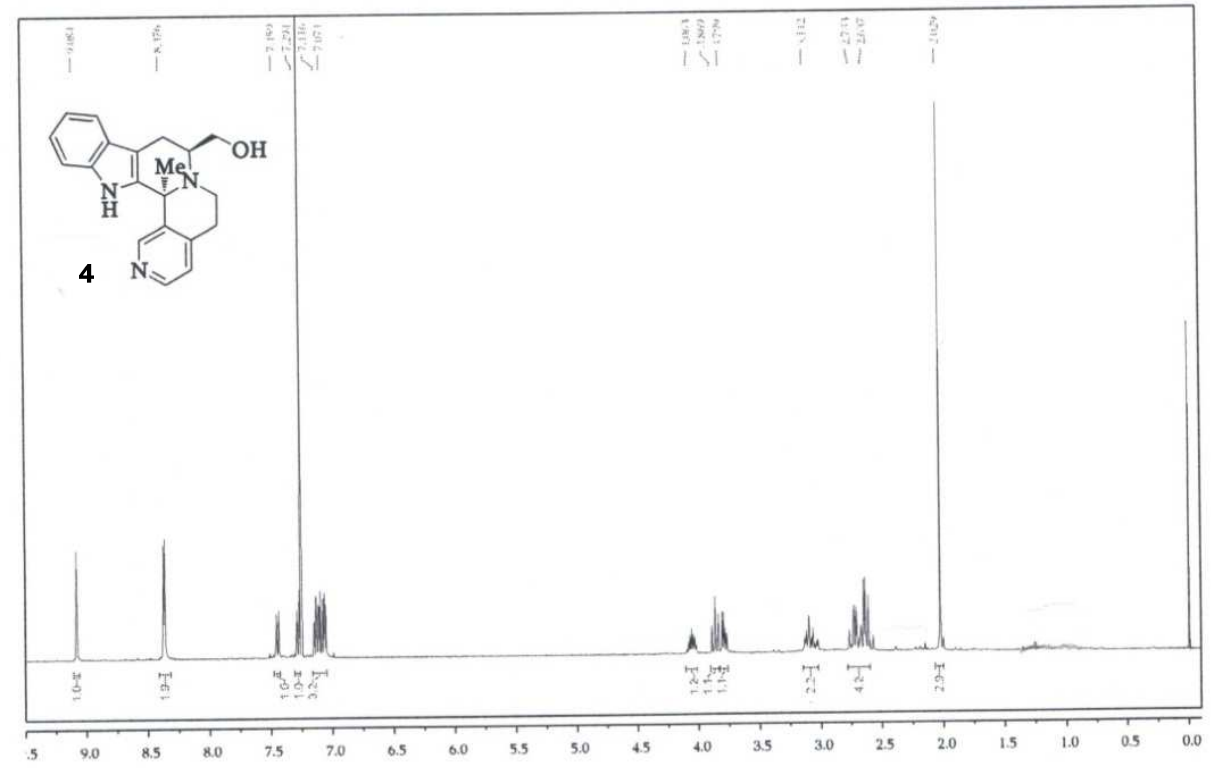

NMR-C ${ }^{13} /$ Mercury-400

$\mathrm{T}=25^{\circ} \mathrm{C} / \mathrm{CDCl}_{3}$

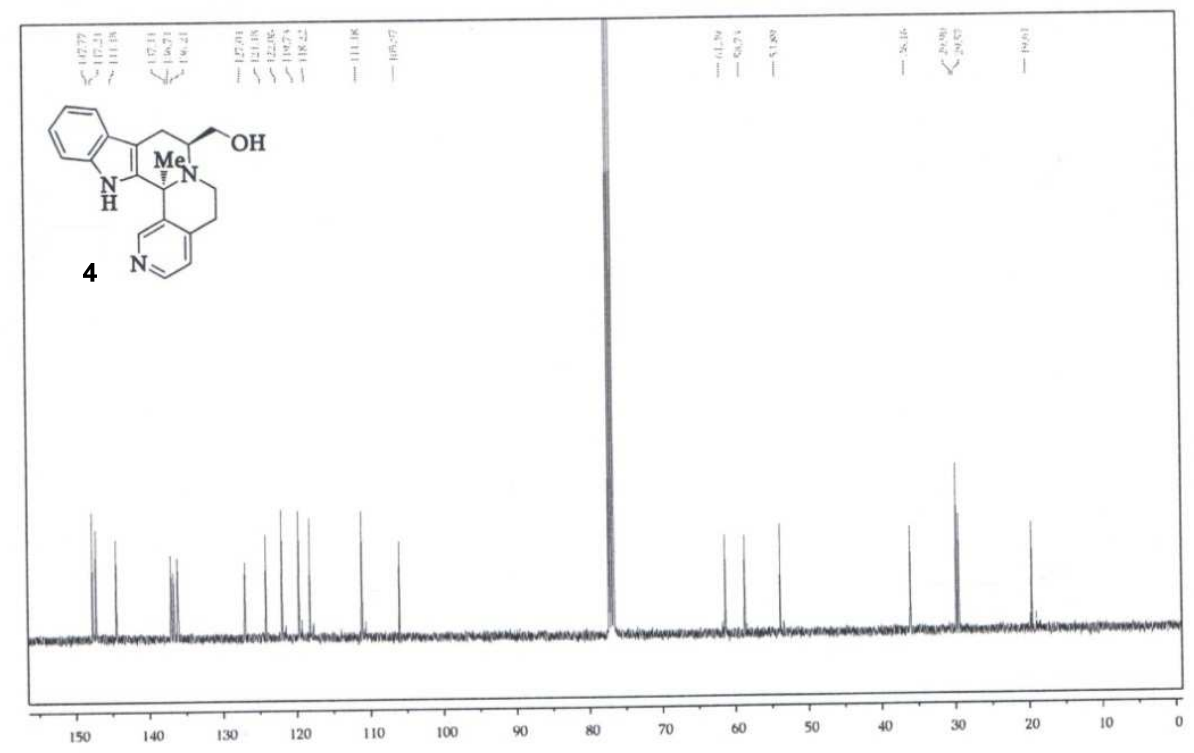


NMR-H $1 /$ Mercury-400

$\mathrm{T}=25^{\circ} \mathrm{C} / \mathrm{CDCl}_{3}$

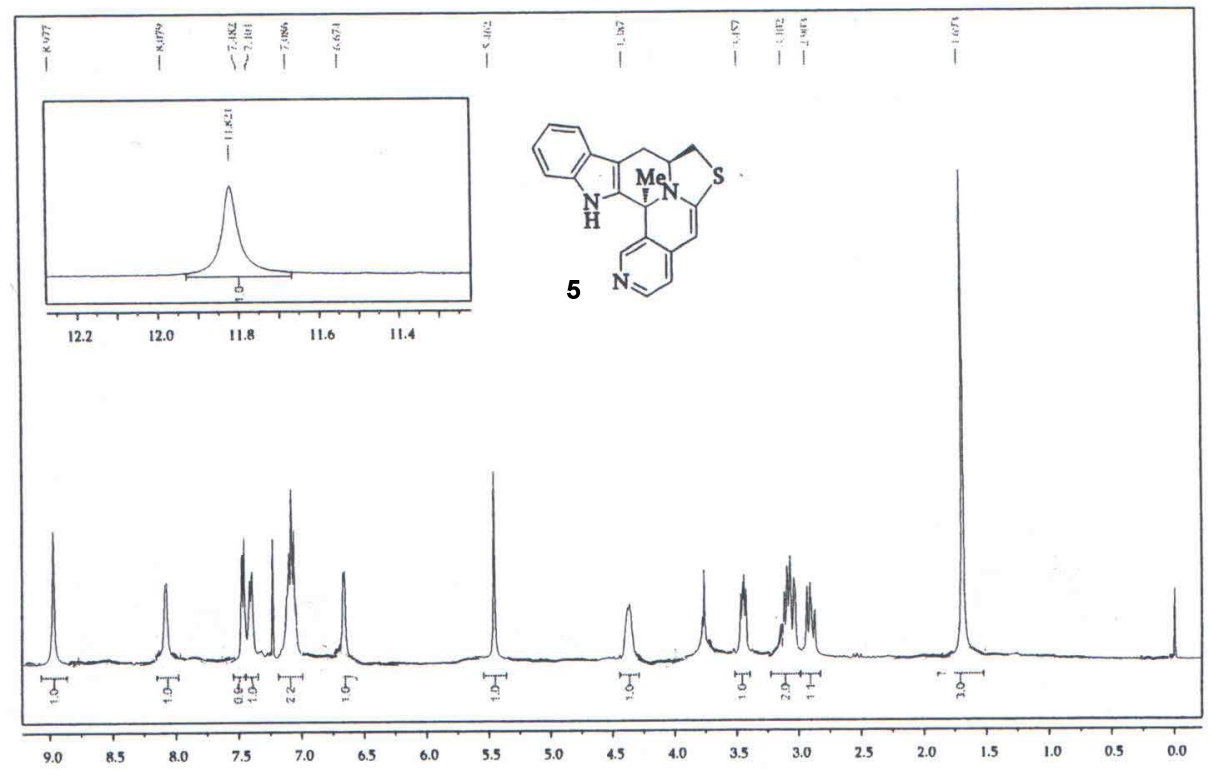

NMR-C ${ }^{13} /$ Gemini-300

$\mathrm{T}=25^{\circ} \mathrm{C} / \mathrm{CDCl}_{3}$

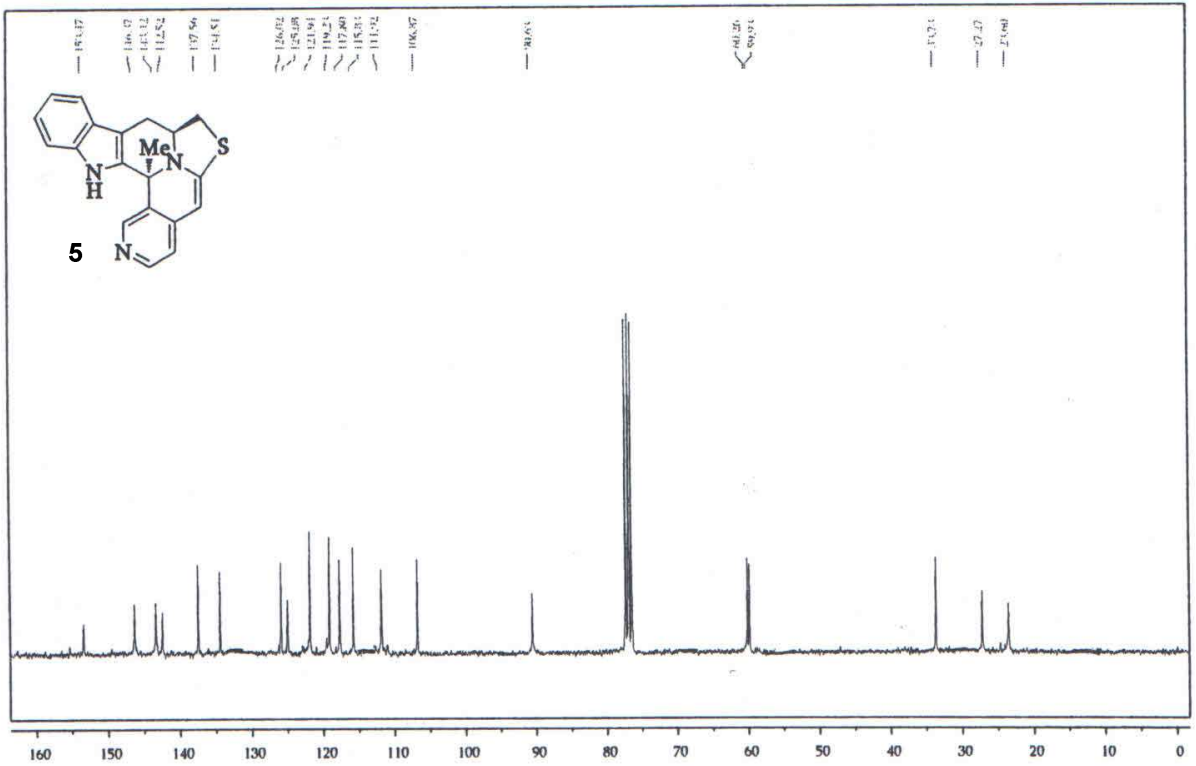


NMR-H $\mathrm{H}^{1} /$ Mercury-400

$\mathrm{T}=25^{\circ} \mathrm{C} / \mathrm{CDCl}_{3}$

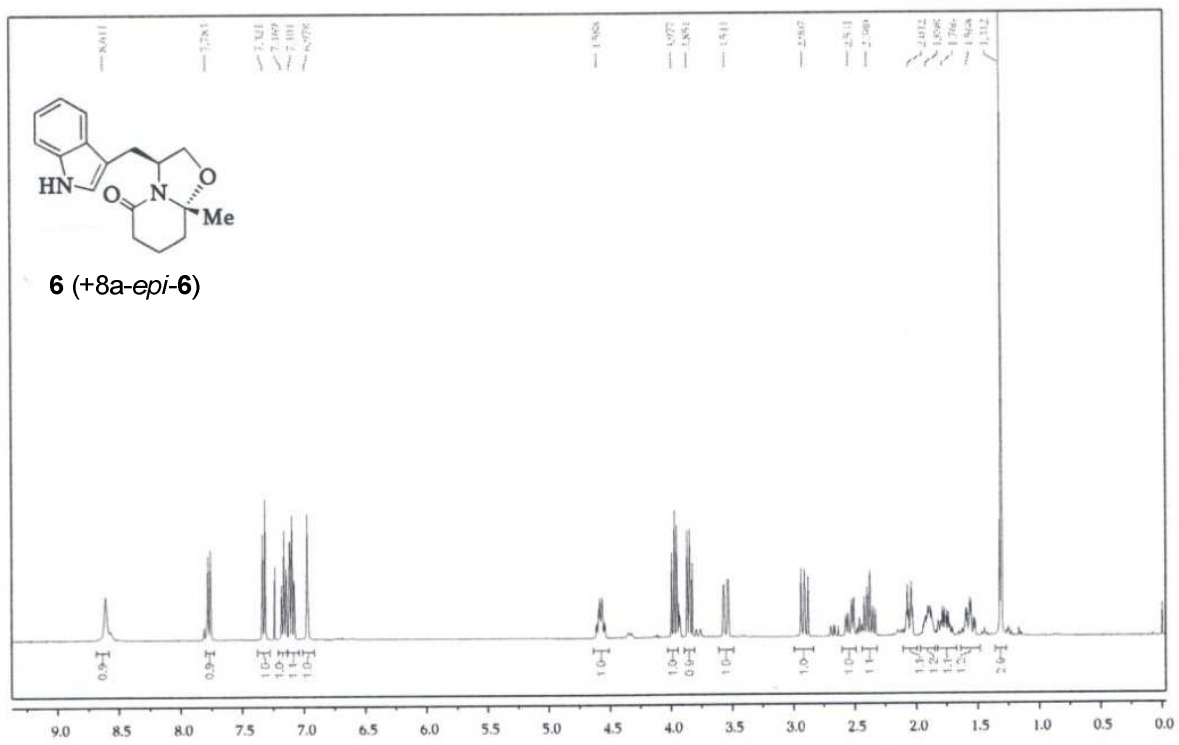

NMR-C ${ }^{13} /$ Mercury-400

$\mathrm{T}=25^{\circ} \mathrm{C} / \mathrm{CDCl}_{3}$

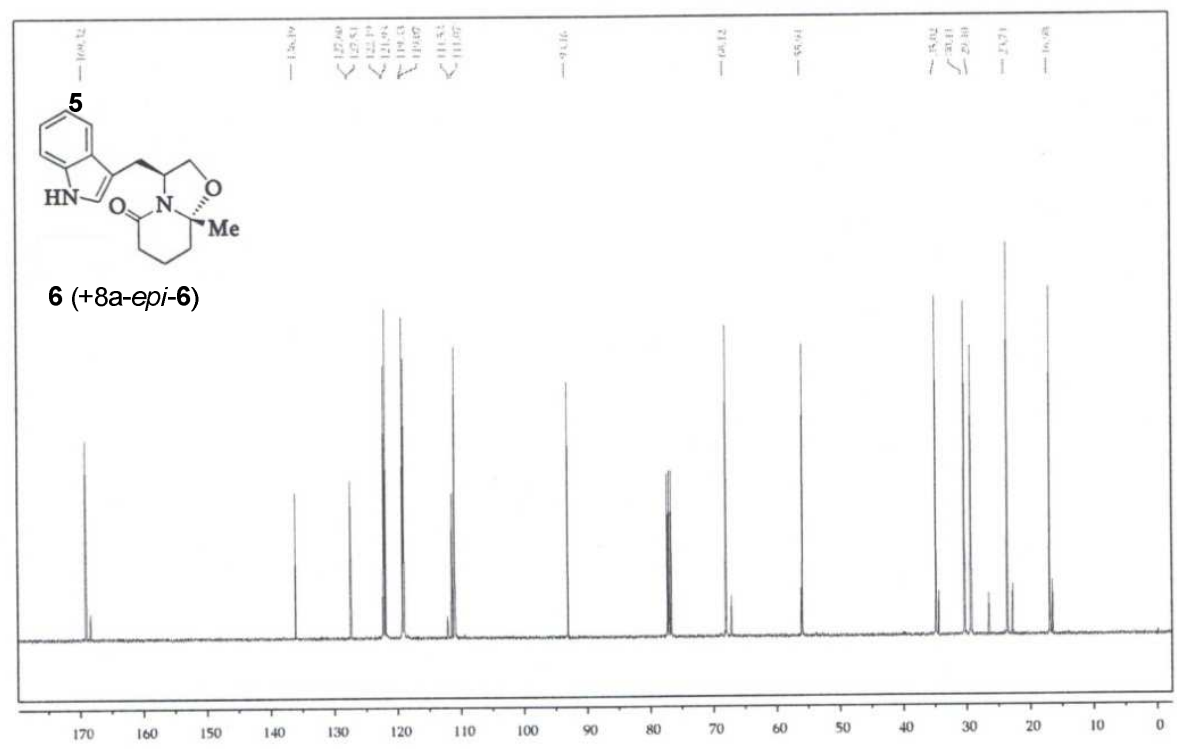


NMR-H ${ }^{1} /$ Mercury-400

$\mathrm{T}=25^{\circ} \mathrm{C} / \mathrm{CDCl}_{3}$

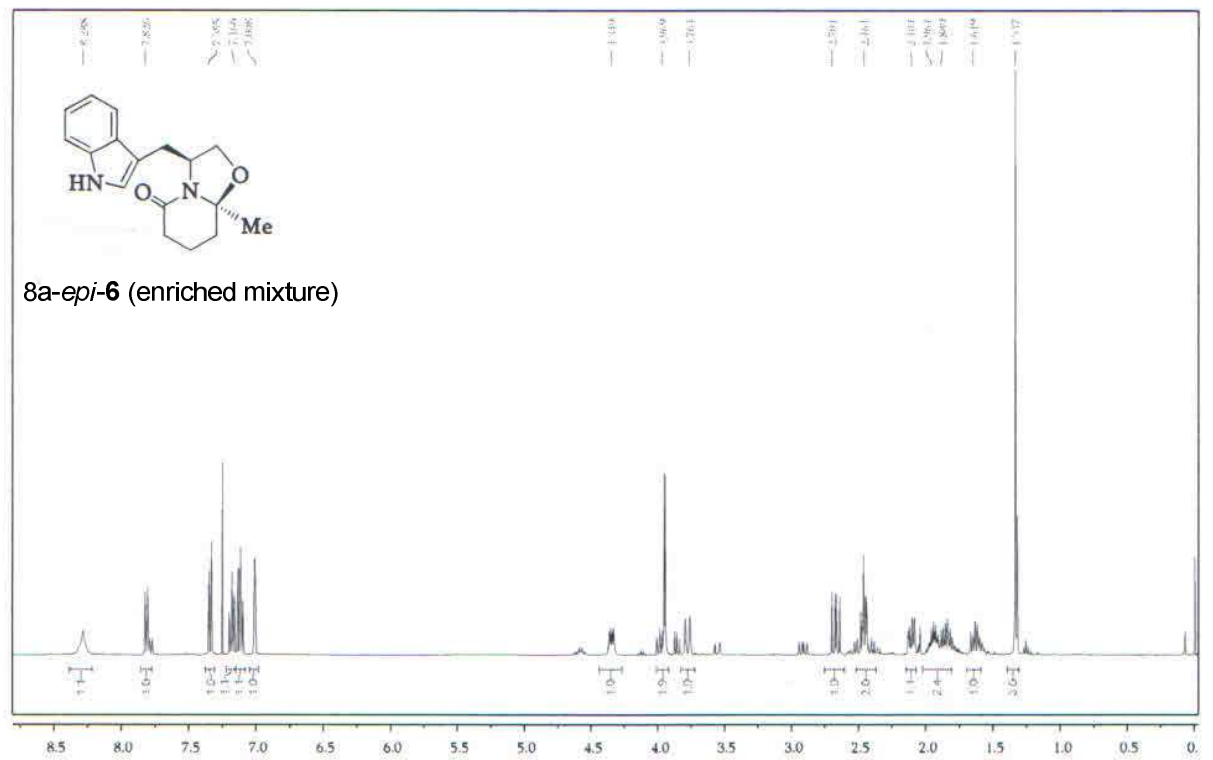

NMR-C ${ }^{13} /$ Mercury-400

$\mathrm{T}=25^{\circ} \mathrm{C} / \mathrm{CDCl}_{3}$

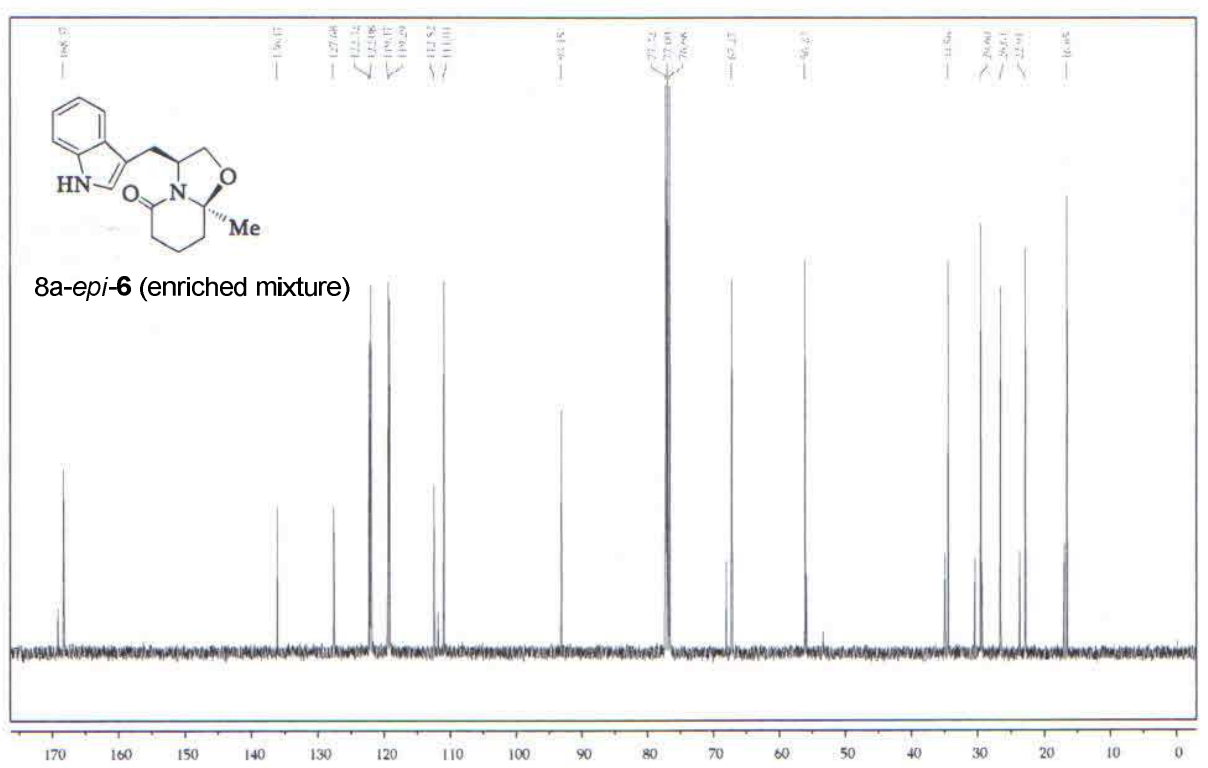


NMR-H'1/Mercury-400

$\mathrm{T}=25^{\circ} \mathrm{C} / \mathrm{CDCl}_{3}$

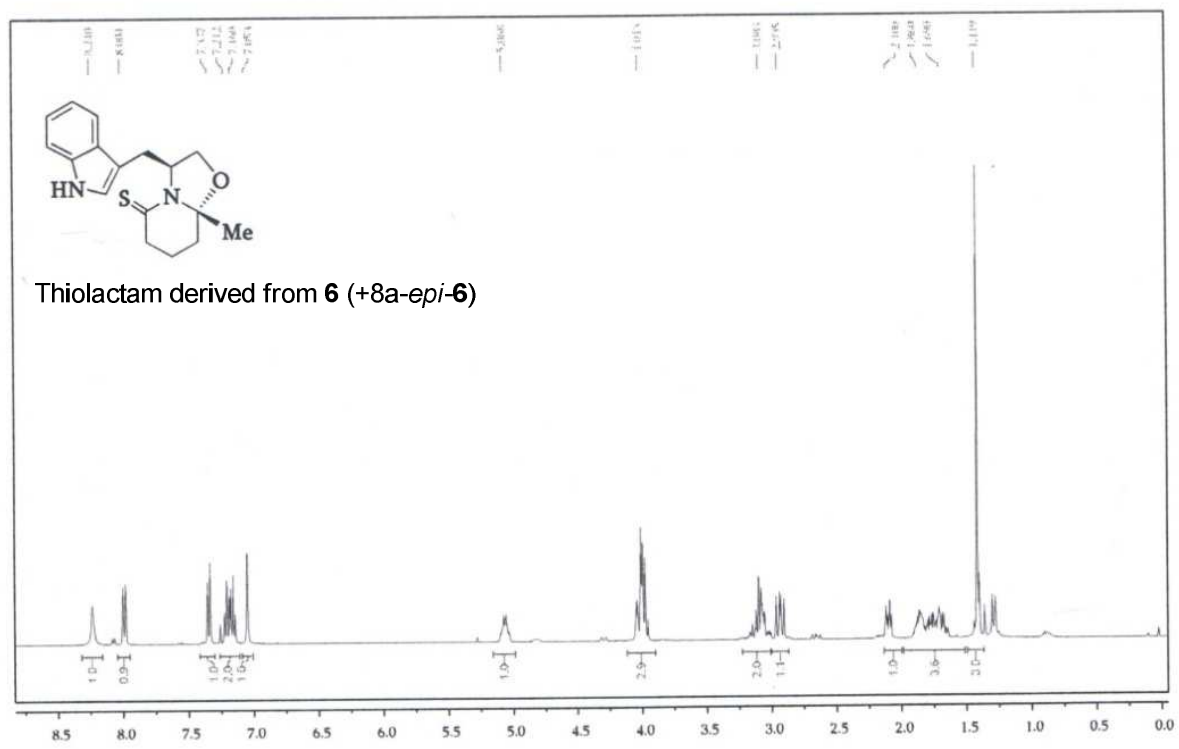

NMR-C ${ }^{13} /$ Mercury-400

$\mathrm{T}=25^{\circ} \mathrm{C} / \mathrm{CDCl}_{3}$

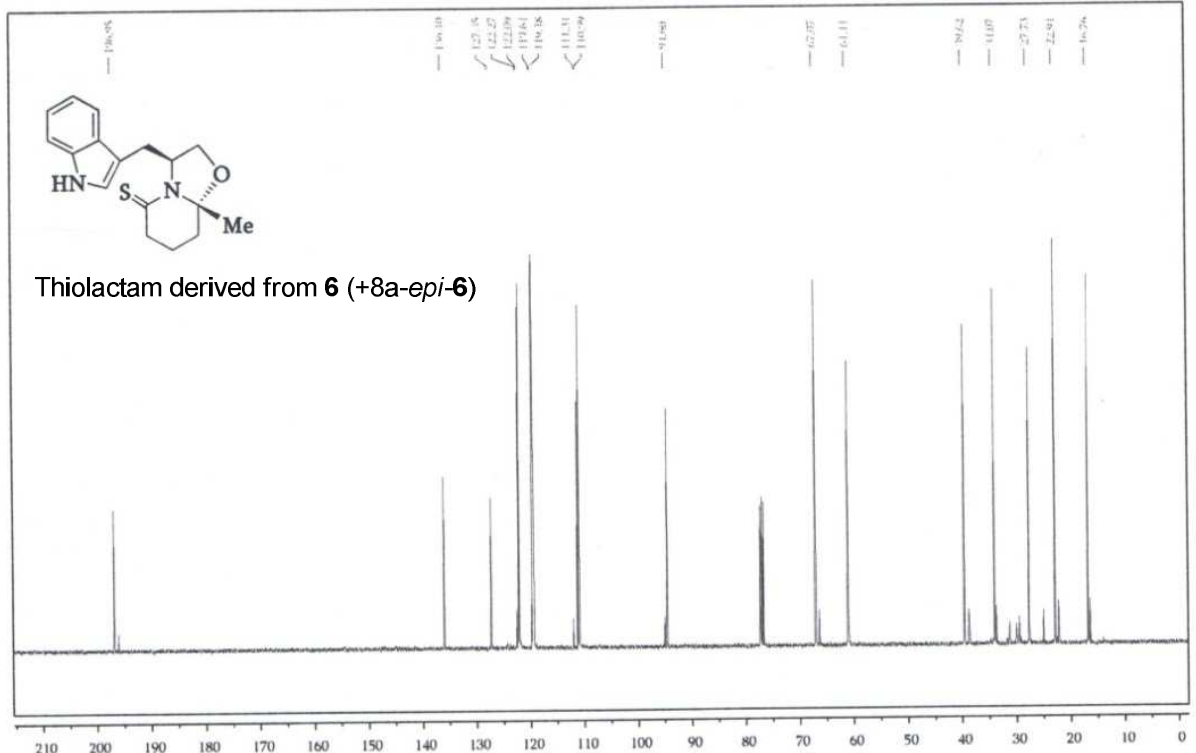


NMR-H $1 /$ Mercury-400

$\mathrm{T}=25^{\circ} \mathrm{C} / \mathrm{CDCl}_{3}$

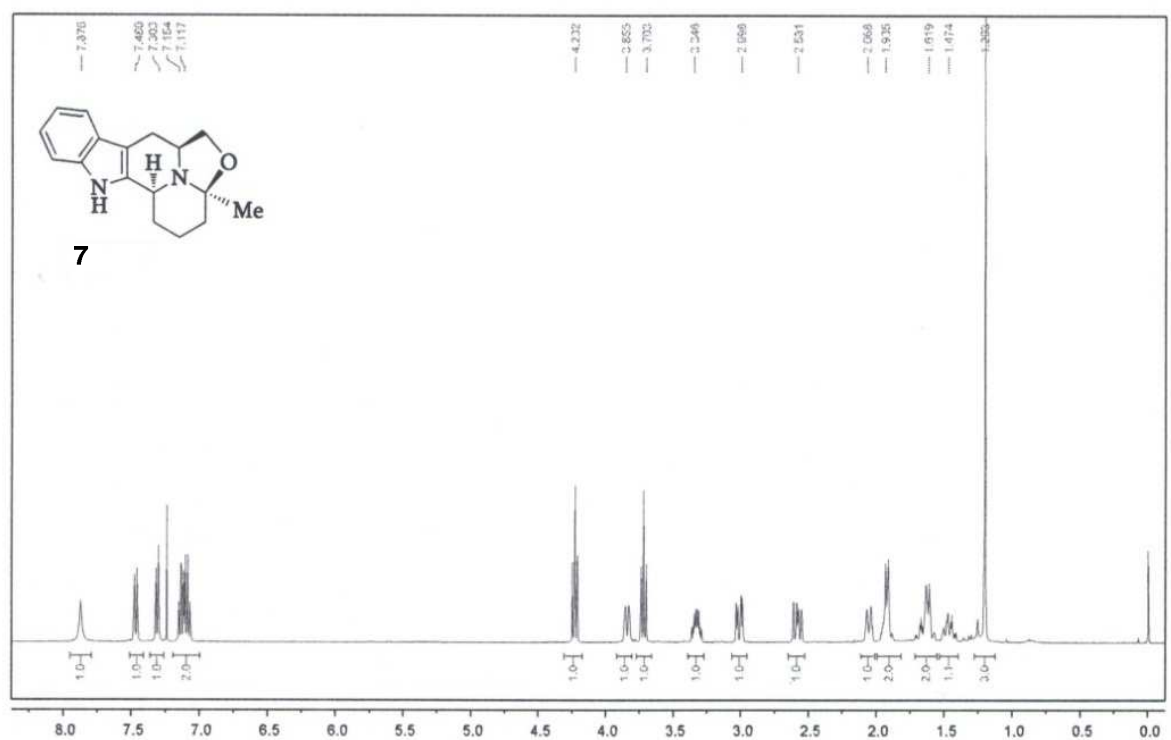

NMR-C ${ }^{13} /$ Mercury-400

$\mathrm{T}=25^{\circ} \mathrm{C} / \mathrm{CDCl}_{3}$

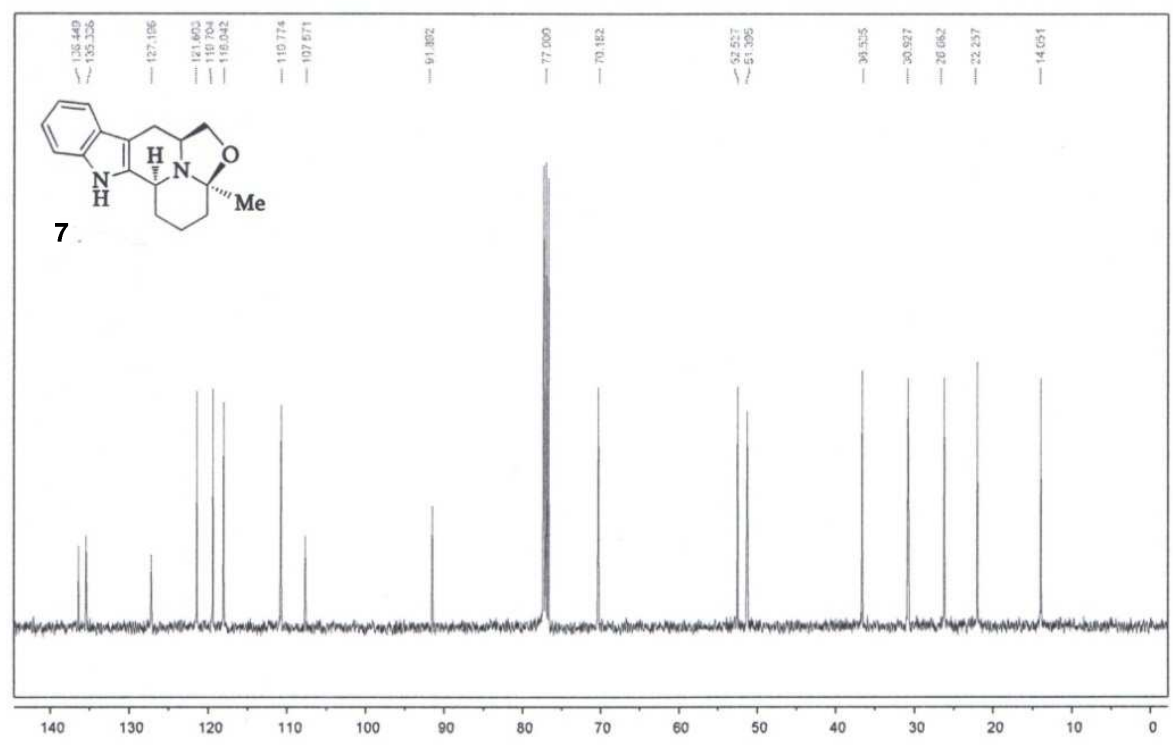


NMR-H ${ }^{1} /$ Mercury-400

$\mathrm{T}=25^{\circ} \mathrm{C} / \mathrm{CDCl}_{3}$

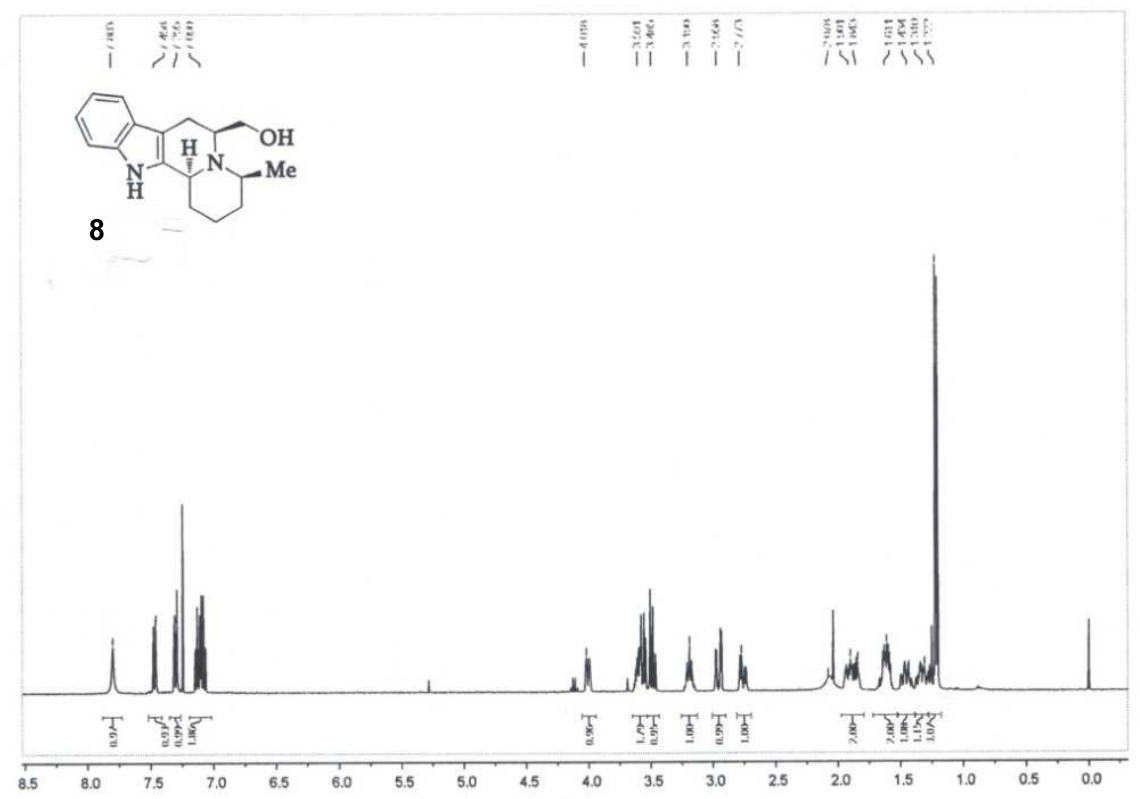

NMR-C ${ }^{13} /$ Mercury-400

$\mathrm{T}=25^{\circ} \mathrm{C} / \mathrm{CDCl}_{3}$

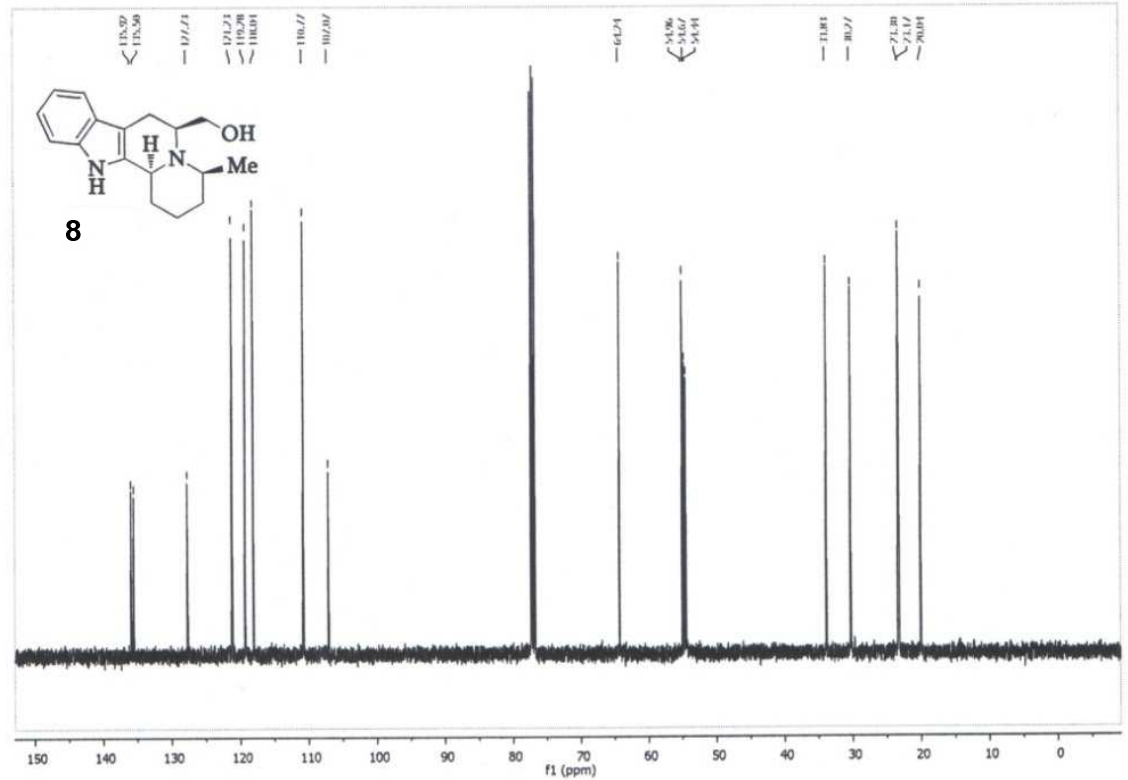


NMR-H $1 /$ Mercury-400

$\mathrm{T}=25^{\circ} \mathrm{C} / \mathrm{CD}_{3} \mathrm{OD}$

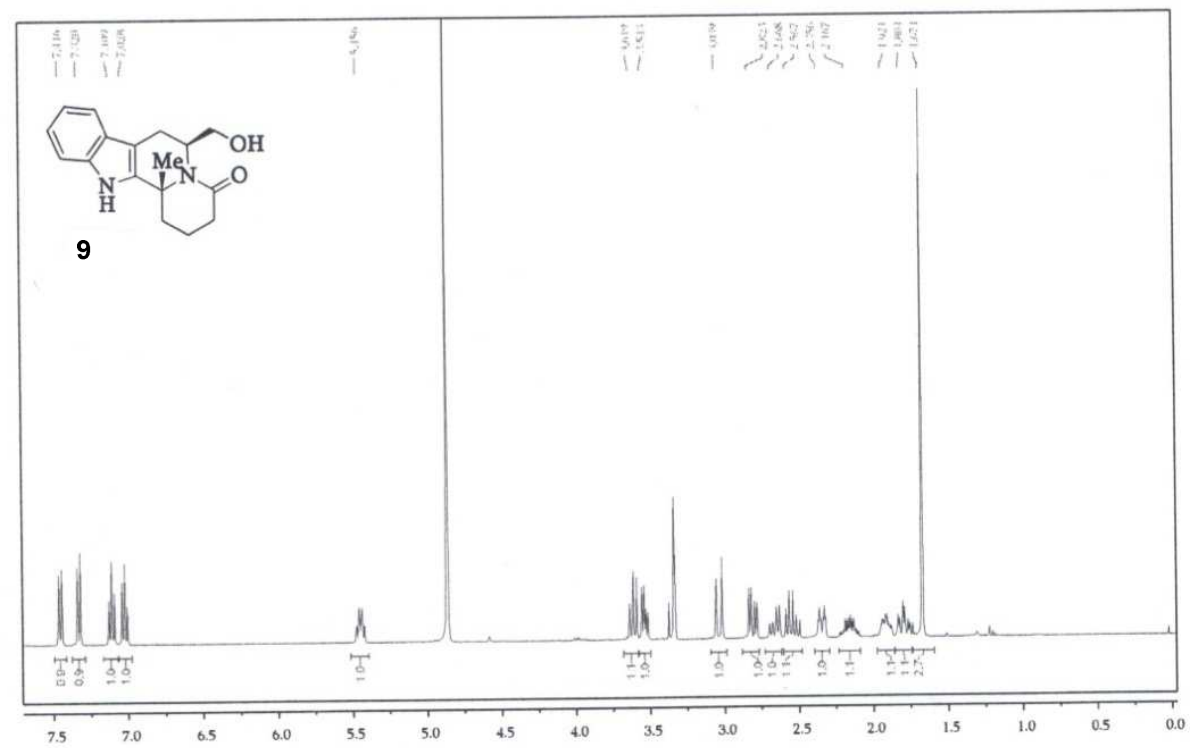

NMR-C ${ }^{13} /$ Mercury-400

$\mathrm{T}=25^{\circ} \mathrm{C} / \mathrm{CD}_{3} \mathrm{OD}$

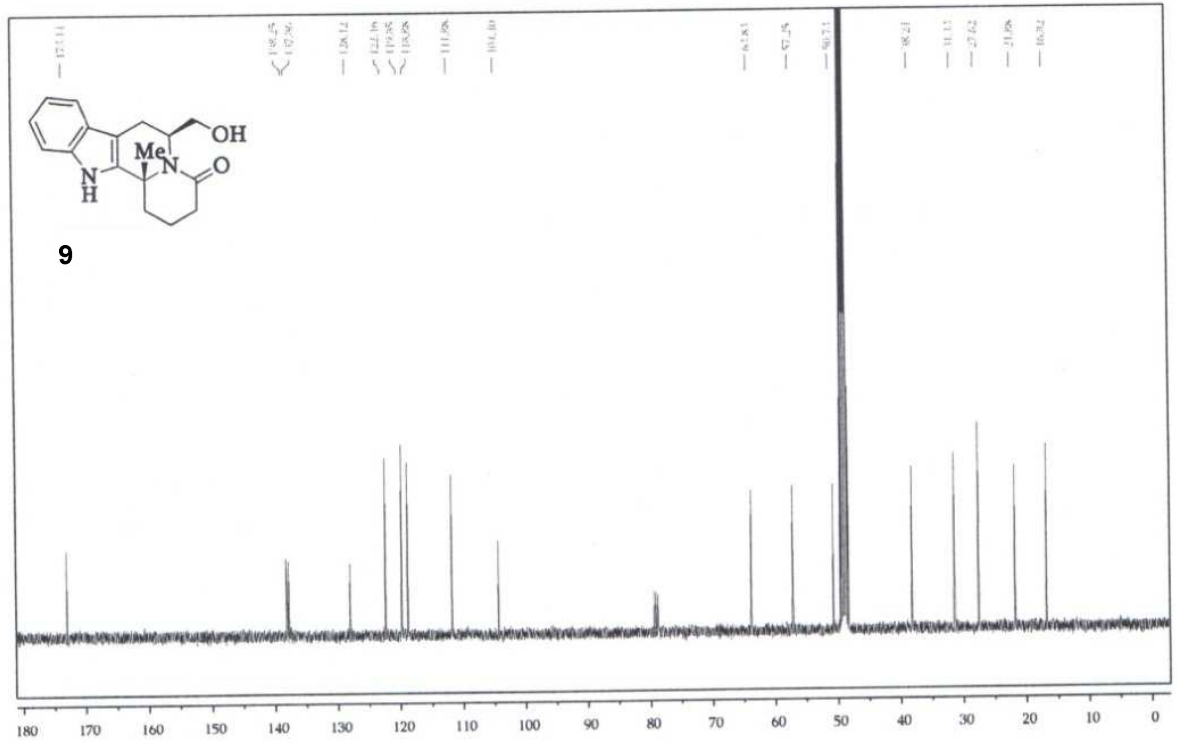


NMR-H ${ }^{1 / M e r c u r y-400 ~}$

$\mathrm{T}=25^{\circ} \mathrm{C} / \mathrm{CD}_{3} \mathrm{OD}$

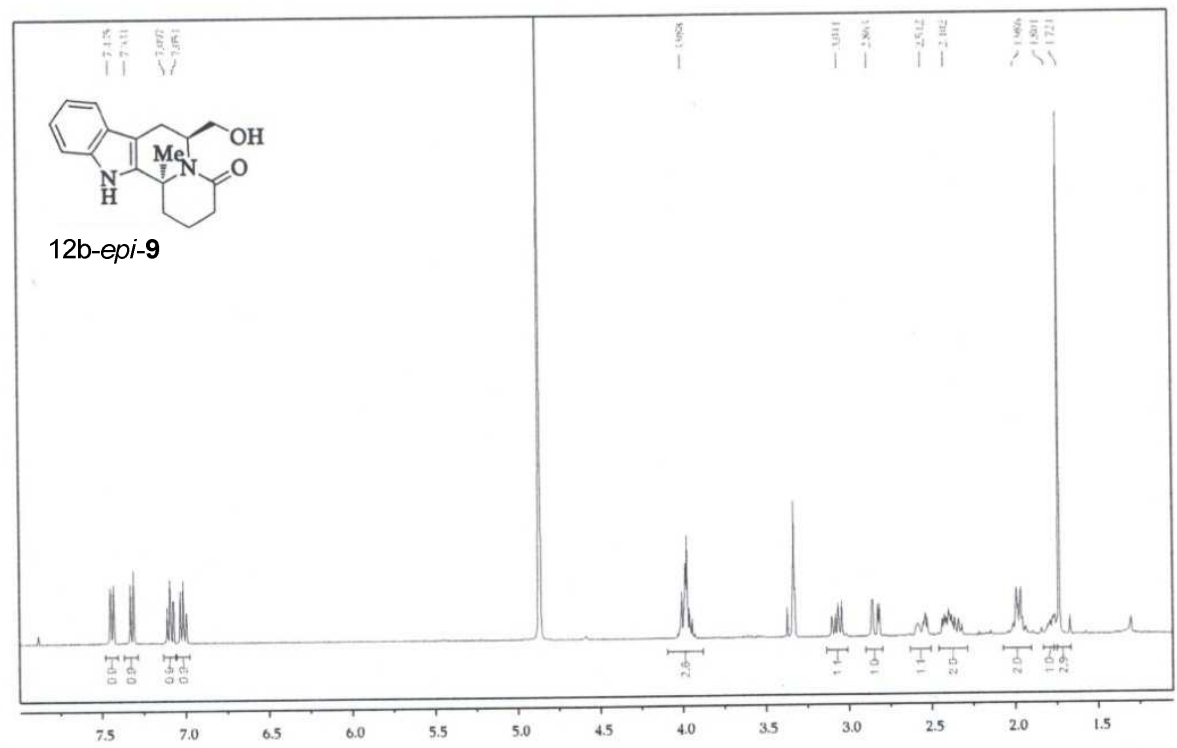

NMR-C ${ }^{13} /$ Mercury-400

$\mathrm{T}=25^{\circ} \mathrm{C} / \mathrm{CD}_{3} \mathrm{OD}$

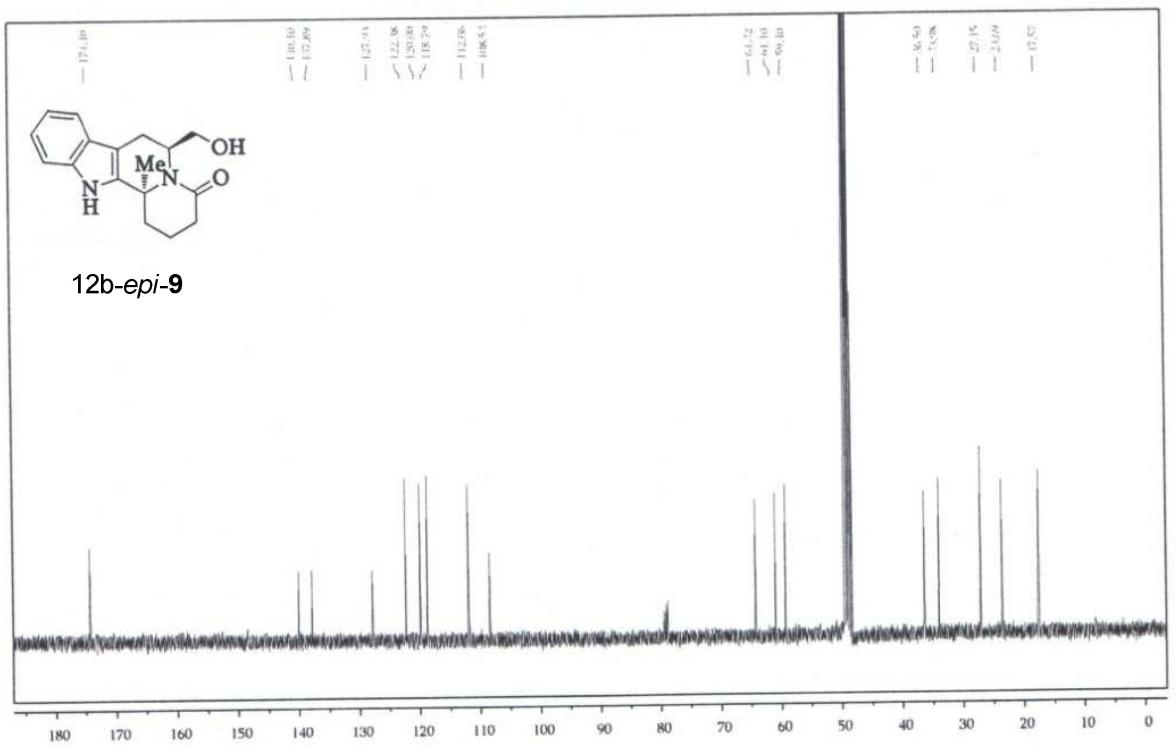


NMR-H ${ }^{1 / M e r c u r y-400 ~}$

$\mathrm{T}=25^{\circ} \mathrm{C} / \mathrm{CDCl}_{3}$

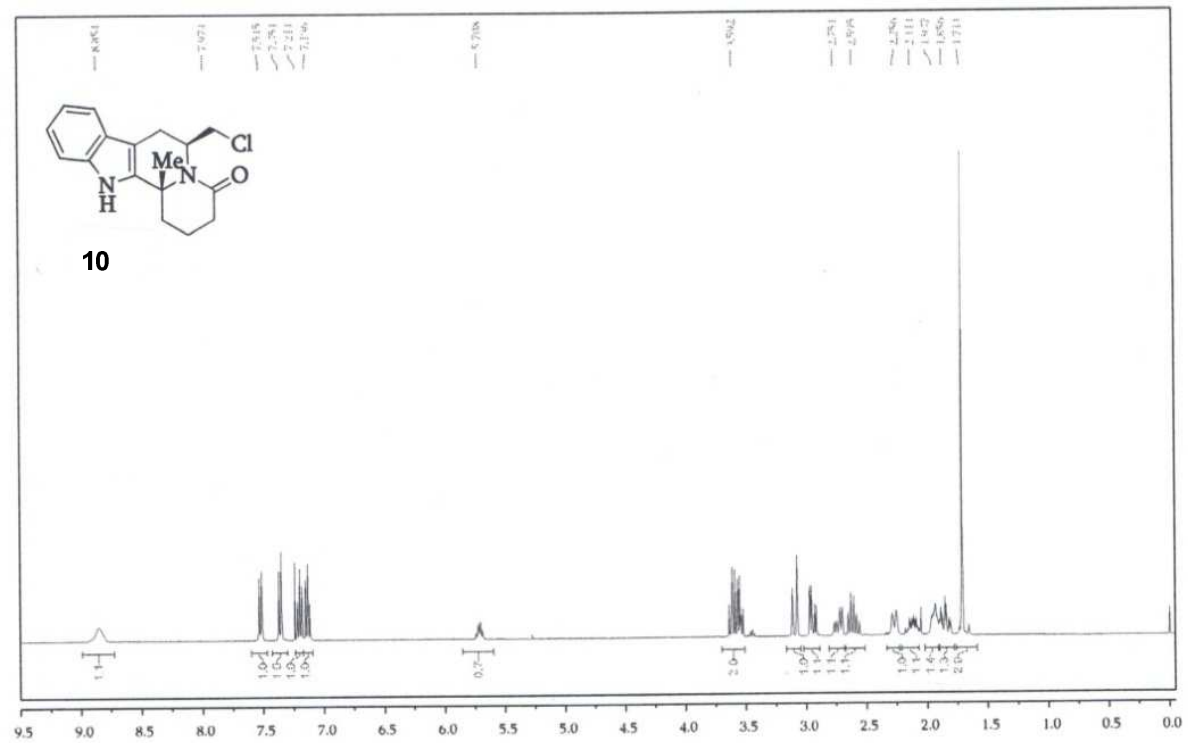

NMR- ${ }^{13} \mathrm{C} /$ Mercury-400

$\mathrm{T}=25^{\circ} \mathrm{C} / \mathrm{CDCl}_{3}$

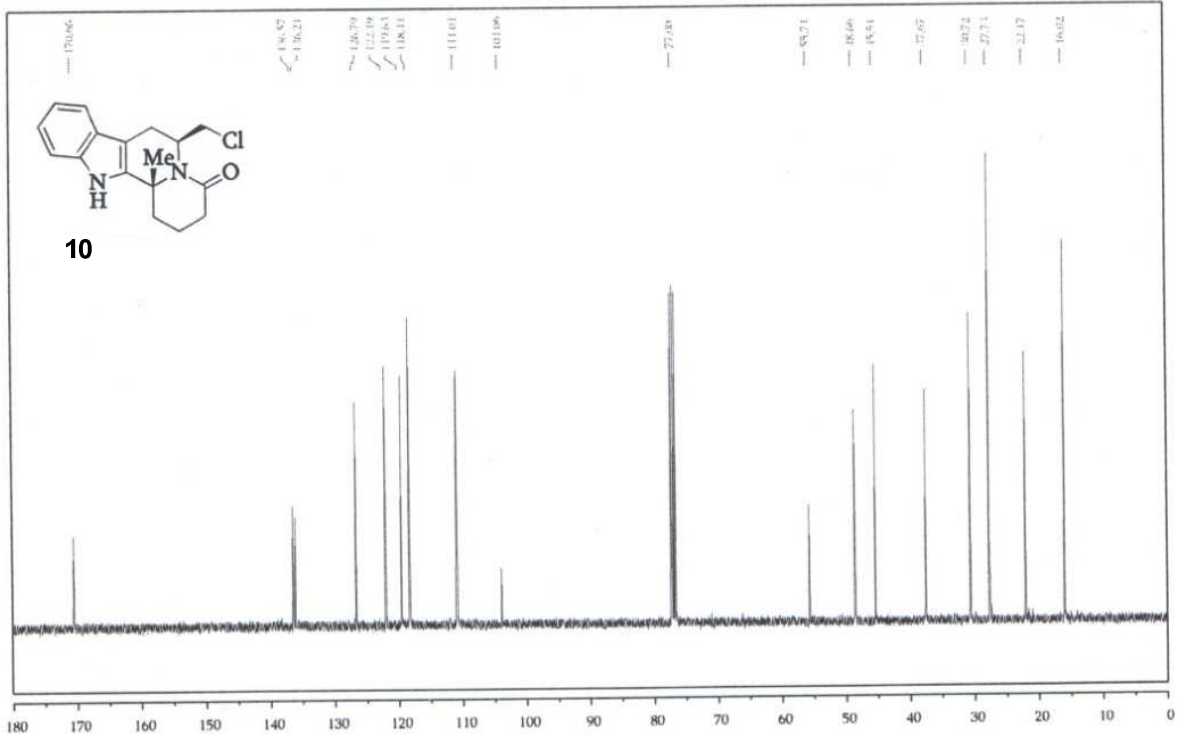




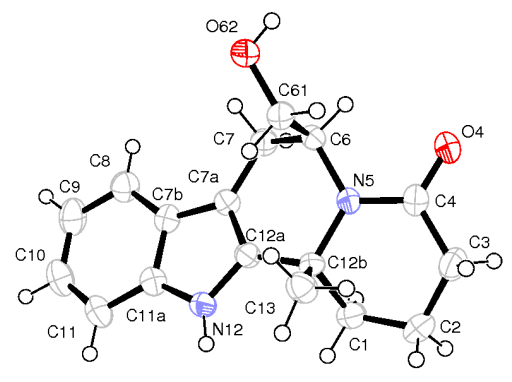

Identification code

Empirical formula

Formula weight

Temperature

Wavelength

Crystal system

Space group

Unit cell dimensions

Volume

$\mathrm{Z}$

Density (calculated)

Absorption coefficient

$\mathrm{F}(000)$

Crystal size

Theta range for data collection

Index ranges

Reflections collected

Independent reflections

Completeness to theta $=24.98^{\circ}$

Refinement method

Data / restraints / parameters

Goodness-of-fit on $\mathrm{F}^{2}$

Final $\mathrm{R}$ indices $[\mathrm{I}>2 \operatorname{sigma}(\mathrm{I})]$

$\mathrm{R}$ indices (all data)

Largest diff. peak and hole
Jb88

$\mathrm{C}_{17} \mathrm{H}_{20} \mathrm{~N}_{2} \mathrm{O}_{2}$

284.35

293(2) K

$0.71073 \AA$

Tetragonal

P 41212

$\begin{array}{ll}\mathrm{a}=10.0470(16) \AA & \alpha=90^{\circ} . \\ \mathrm{b}=10.0470(18) \AA & \beta=90^{\circ} . \\ \mathrm{c}=28.929(6) \AA & \gamma=90^{\circ} .\end{array}$

2920.2(9) $\AA^{3}$

8

$1.294 \mathrm{Mg} / \mathrm{m}^{3}$

$0.086 \mathrm{~mm}^{-1}$

1216

$0.27 \times 0.27 \times 0.12 \mathrm{~mm}^{3}$

2.15 to $24.98^{\circ}$.

$-7<=\mathrm{h}<=8,0<=\mathrm{k}<=11,0<=\mathrm{l}<=34$

5841

2566

$100.0 \%$

Full-matrix least-squares on $\mathrm{F}^{2}$

2566 / 0 / 192

1.054

$\mathrm{R} 1=0.0428, \mathrm{wR} 2=0.0814$

$\mathrm{R} 1=0.0688, \mathrm{wR} 2=0.0893$

0.124 and -0.118 e. $\AA^{-3}$ 


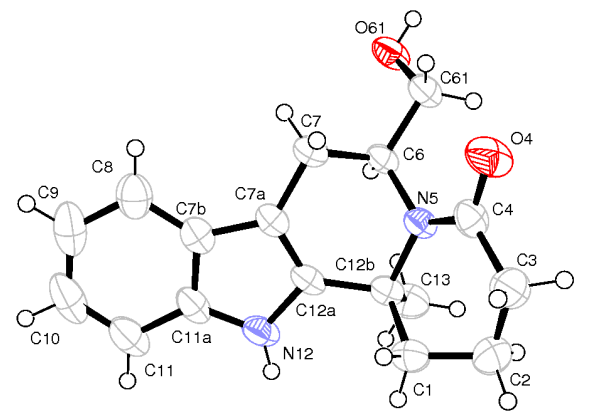

Formula weight

Temperature

Wavelength

Crystal system

Space group

Unit cell dimensions

Volume

$\mathrm{Z}$

Density (calculated)

Absorption coefficient

$\mathrm{F}(000)$

Crystal size

Theta range for data collection

Index ranges

Reflections collected

Independent reflections

Completeness to theta $=26.97^{\circ}$

Max. and min. transmission

Refinement method

Data / restraints / parameters

Goodness-of-fit on $\mathrm{F}^{2}$

Final $\mathrm{R}$ indices [I $>2 \operatorname{sigma}(\mathrm{I})]$

$\mathrm{R}$ indices (all data)

Largest diff. peak and hole
Jb89

$\mathrm{C}_{17} \mathrm{H}_{20} \mathrm{~N}_{2} \mathrm{O}_{2}$

284.35

294(2) K

$0.71073 \AA$

Orthorhombic

P 212121

$\begin{array}{ll}\mathrm{a}=10.164(2) \AA & \alpha=90^{\circ} . \\ \mathrm{b}=10.178(4) \AA & \beta=90^{\circ} . \\ \mathrm{c}=14.373(4) \AA & \gamma=90^{\circ} .\end{array}$

$1486.9(8) \AA^{3}$

4

$1.270 \mathrm{Mg} / \mathrm{m}^{3}$

$0.084 \mathrm{~mm}^{-1}$

608

$0.42 \times 0.42 \times 0.21 \mathrm{~mm}^{3}$

2.45 to $26.97^{\circ}$.

$0<=\mathrm{h}<=12,0<=\mathrm{k}<=12,0<=\mathrm{l}<=18$

1982

1848

$100.0 \%$

0.9826 and 0.9656

Full-matrix least-squares on $\mathrm{F}^{2}$

1848 / 0 / 192

1.029

$\mathrm{R} 1=0.0410, \mathrm{wR} 2=0.0852$

$\mathrm{R} 1=0.0662, \mathrm{wR} 2=0.0935$

0.133 and -0.139 e. $\AA^{-3}$ 